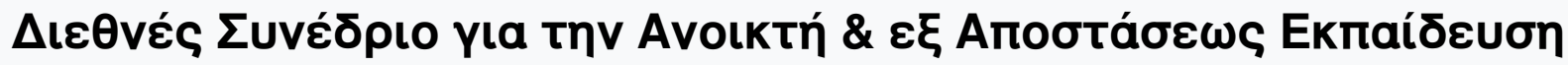

Tón. 6, Ap. 1A (2011)

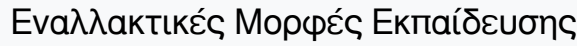

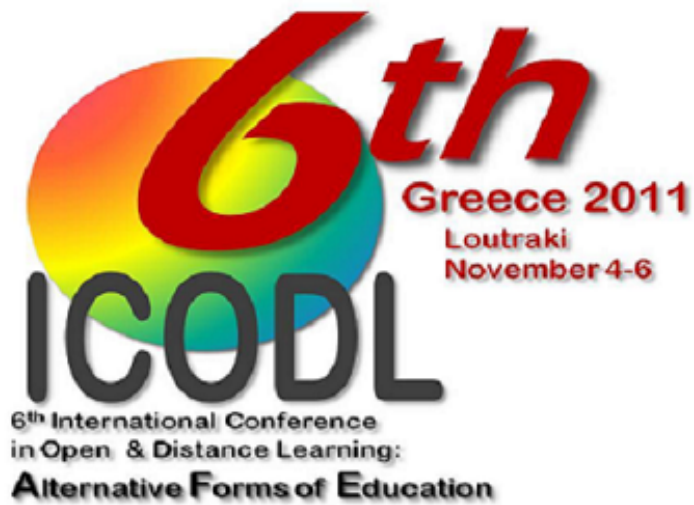

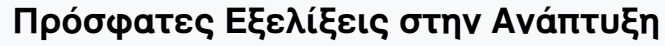

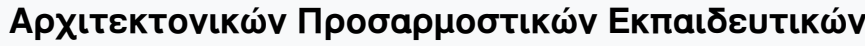

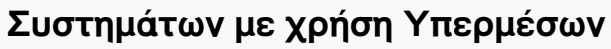

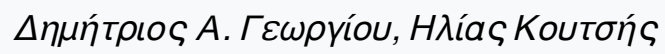
doi: $10.12681 /$ icodl. 692

TOMOEA

PART/MEPOE A 


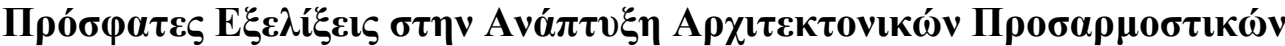

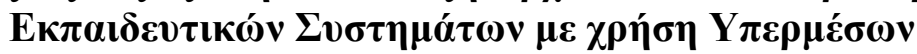

\section{Recent Contributions to the Development of Adaptive Educational Hypermedia Systems Architectures}

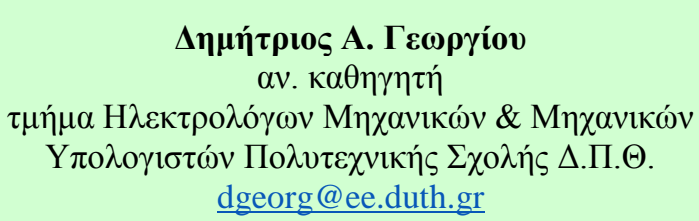

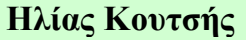

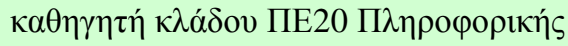

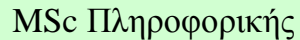 \\ ilias_k@yahoo.com
}

\begin{abstract}
The asynchronous education is a challenge for the development of new methods and troubleshooting in areas such as Human-Computer Interaction. The objective is to develop software that would incorporate all the advantages of an experienced and well educated human tutor, using the capabilities of modern Internet and computer systems that make up. To achieve such an objective one requires the use of modern learning theories, cognitive psychology, diagnostic methods and techniques of instruction, from the area of science education. It is also necessary to apply methodologies that allow us to transform qualitative characteristics of the students, or the teaching material and assessments in purely quantitative terms, that is manageable by the machines. Such methods may be obtained from the fuzzy logic, by Bayesian models or neural networks that allow the handling of new situations on the basis of previous experience. Many scientific disciplines, contribute to the development of new methods in Adaptive Educational Hypermedia Systems. These systems are the true heirs of Learning Management Systems of the 90s and Intelligent Tutoring Systems of the 21 st century's first decade. The main feature here is the concept of adaptivity. This property, on which the Adaptive WEB rely on, asks for new requirements for any single AEHS's element. For example, adaptive retrieve of learning objects from their repositories, requires the use of semantic markup that accompany each learning object. Below, we will try to describe the research activity, which takes place in the Department of Electrical and Computer Engineering at the Democritus University of Thrace, School of Engineering.
\end{abstract}

\section{Пєрі́ $\eta \psi \eta$}

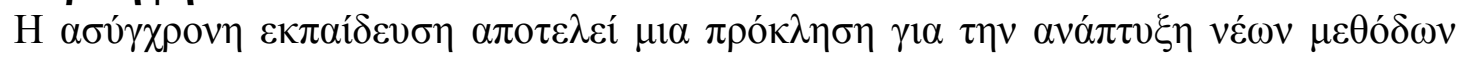

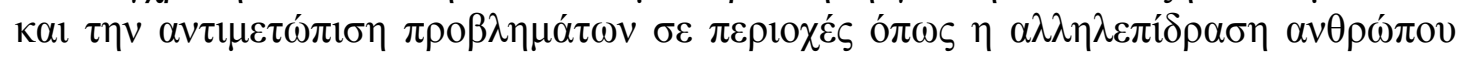

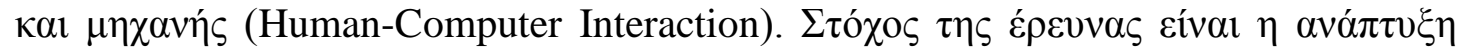

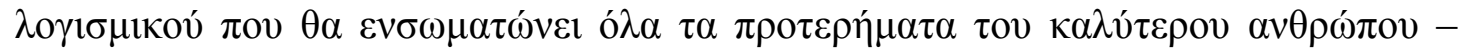

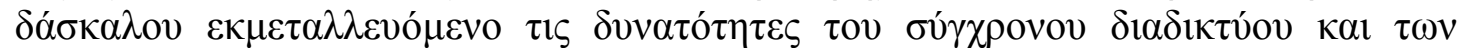

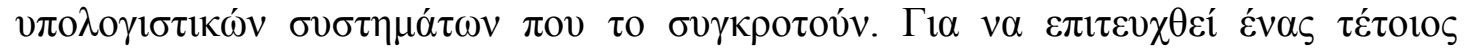

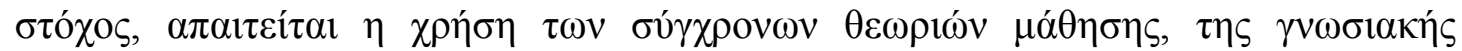

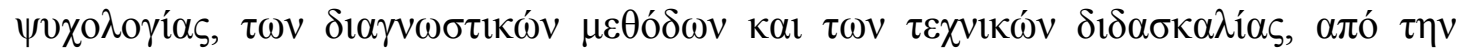

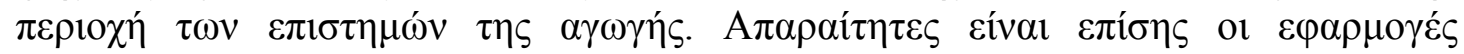

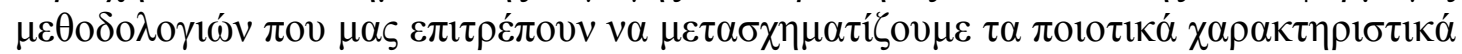

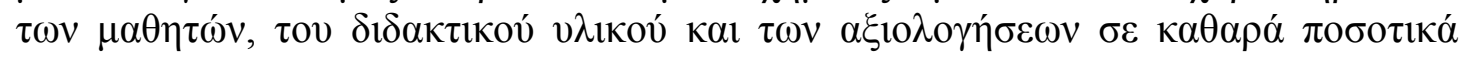




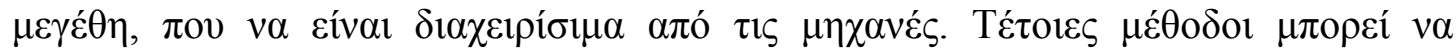

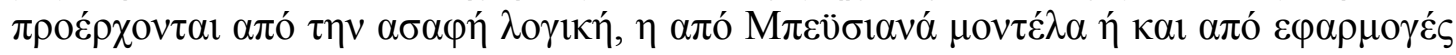

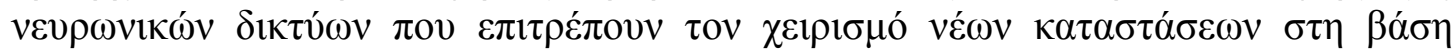

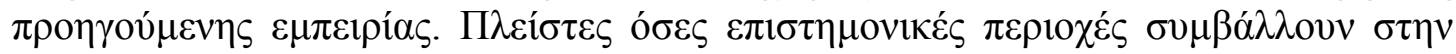

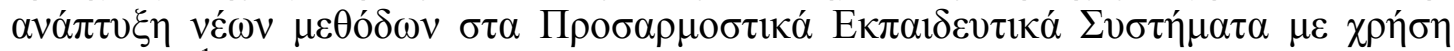

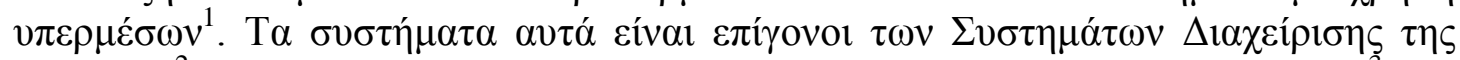

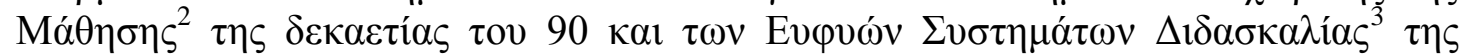

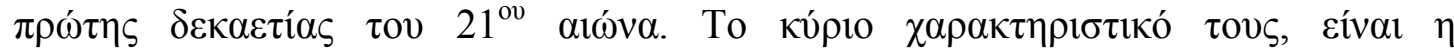

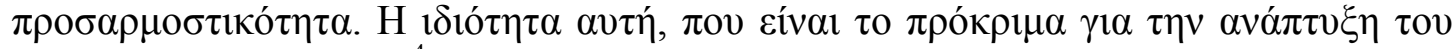

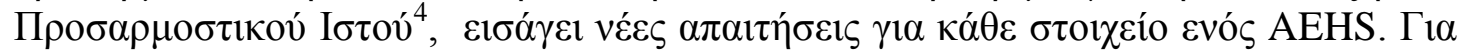

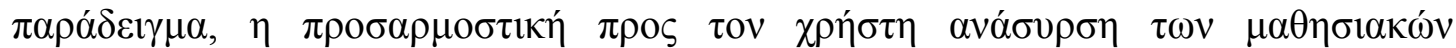

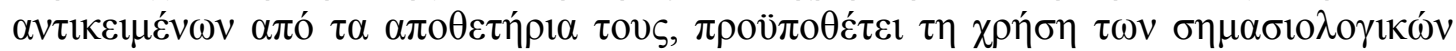

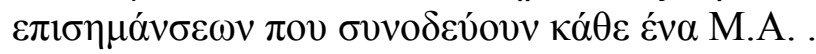

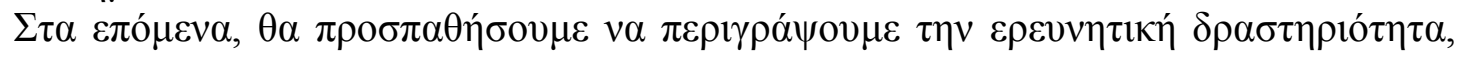

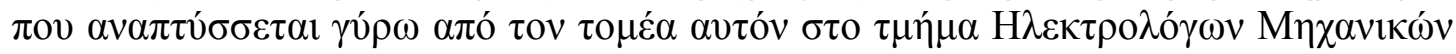

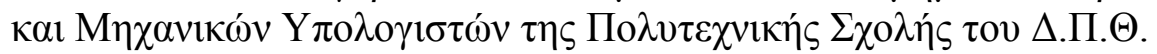

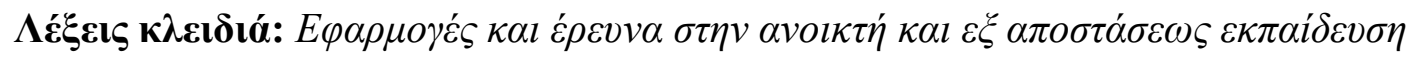

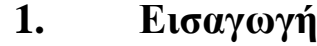

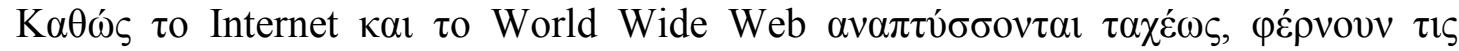

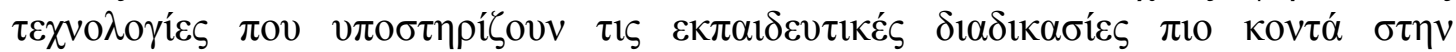

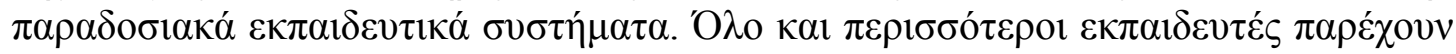

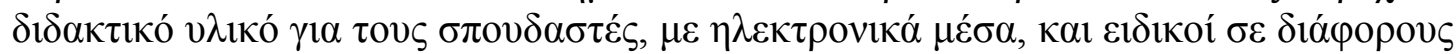

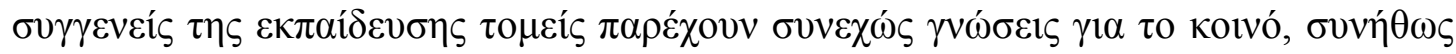

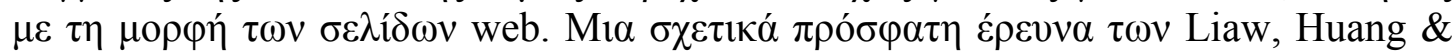

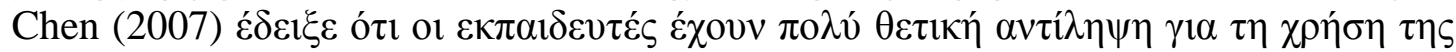

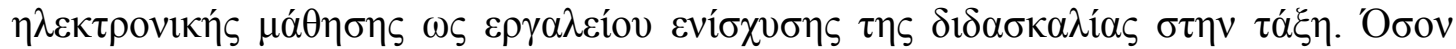

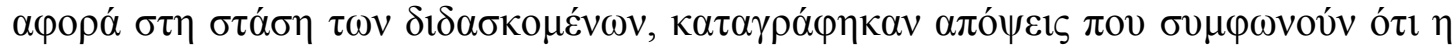

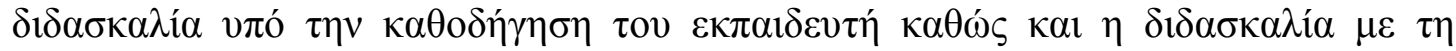

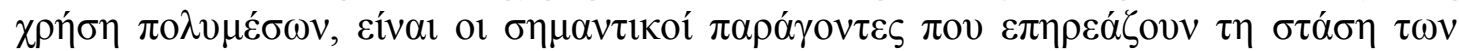

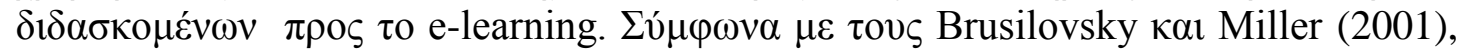

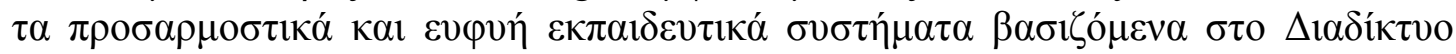

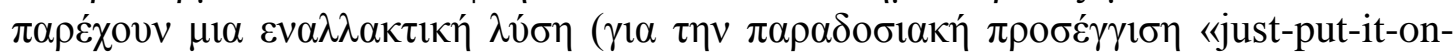

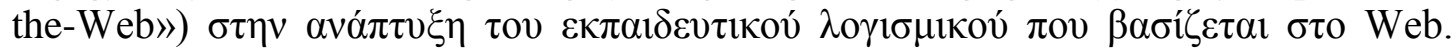

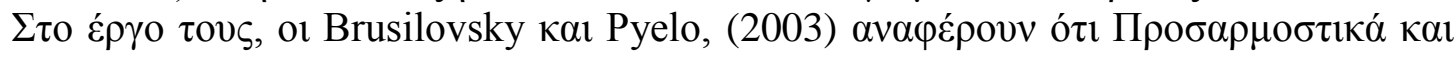

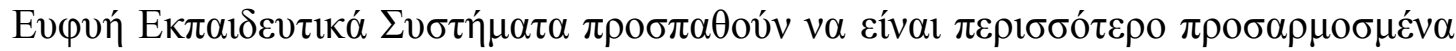

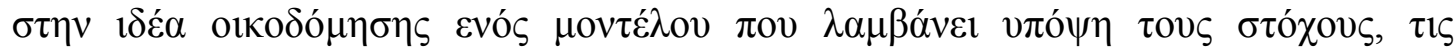

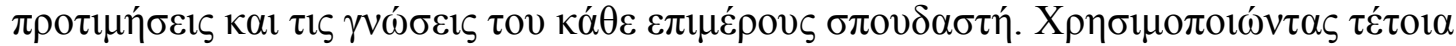

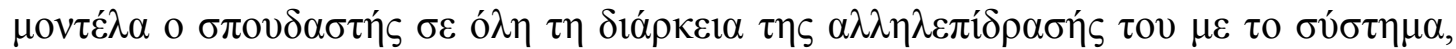

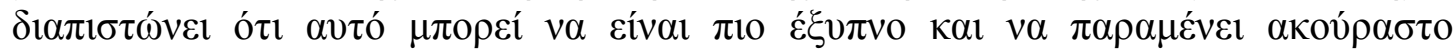

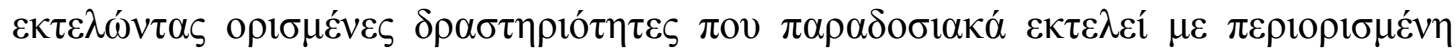

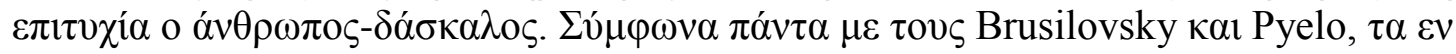

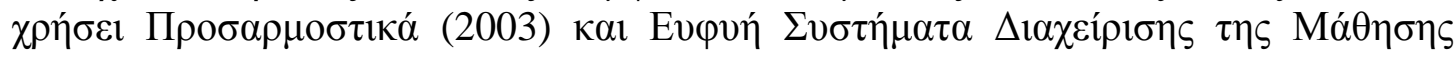

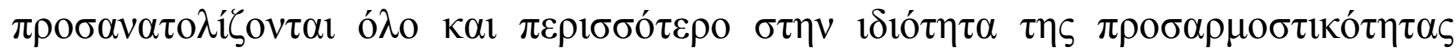

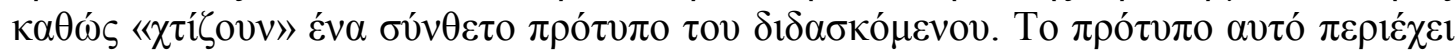

\footnotetext{
${ }^{1}$ Adaptive Educational Hypermedia Systems (AEHS)

${ }^{2}$ Learning Management Systems (LMS)

${ }^{3}$ Intelligent Tutoring Systems (ITS)

${ }^{4}$ Adaptive WEB
} 


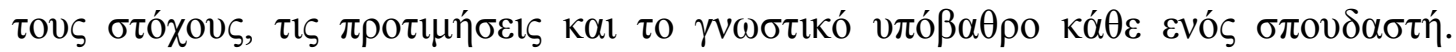

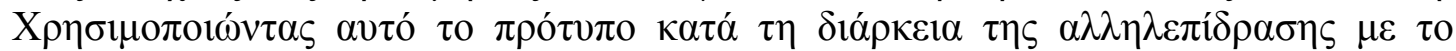

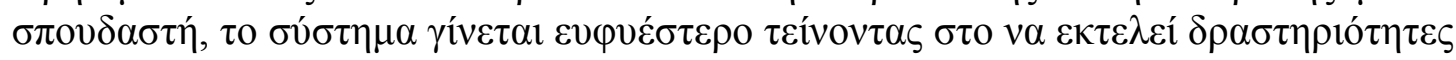

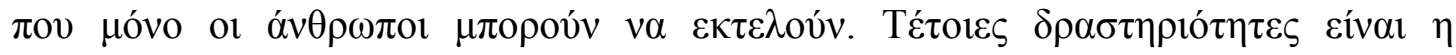

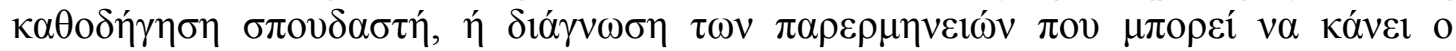

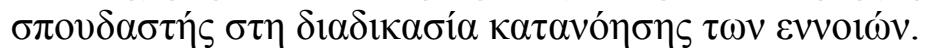

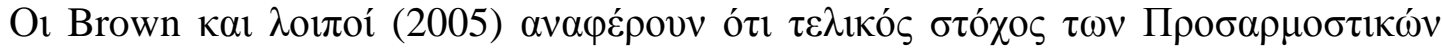

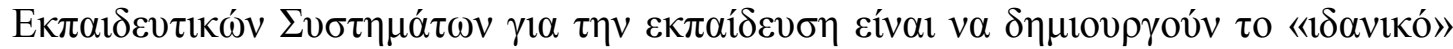
online $\mu \alpha \dot{\theta \eta \eta \mu \alpha ~ \gamma l \alpha ~ \kappa \alpha ́ \theta \varepsilon ~ \chi \rho \eta ́ \sigma \tau \eta ~ \tau o v ~-~ \alpha \xi ı \pi o เ \omega ́ v \tau \alpha \varsigma ~ \varepsilon ́ v \alpha ~ \kappa o เ v o ́ ~ \sigma u ́ v o \lambda o ~ \pi o ́ \rho \omega v ~}$

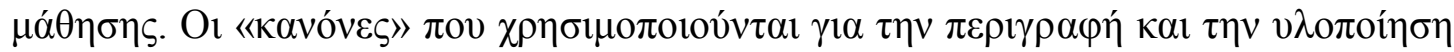

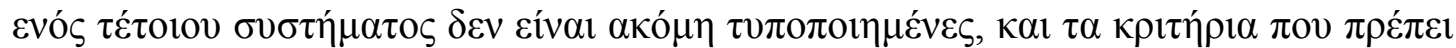

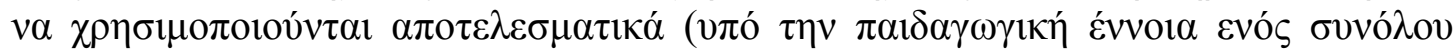

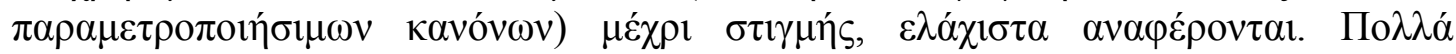

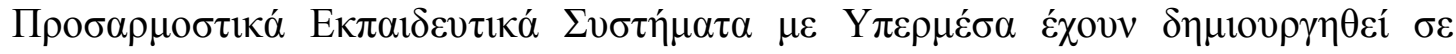

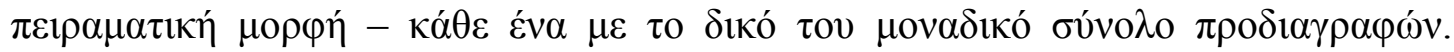

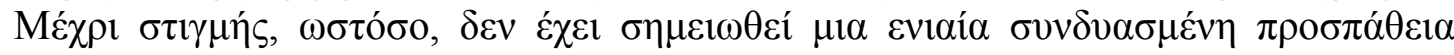

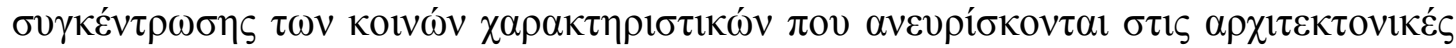

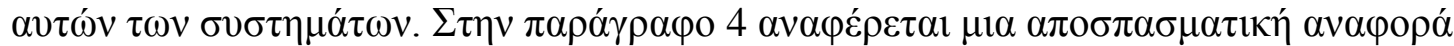

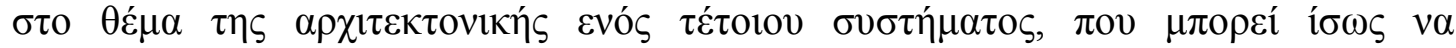

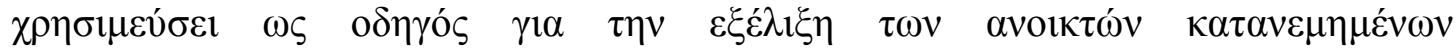

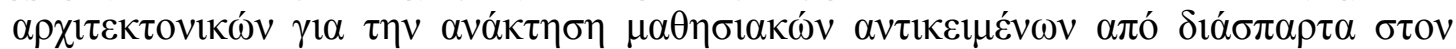

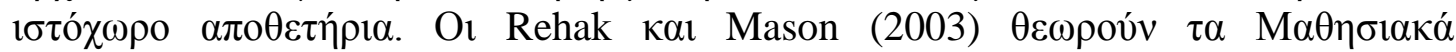

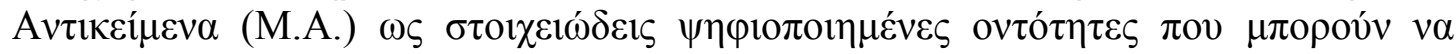

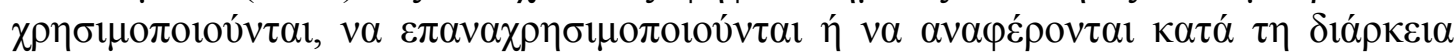

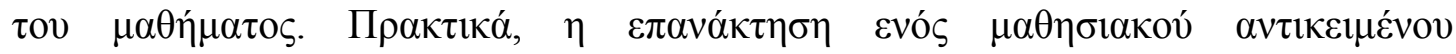

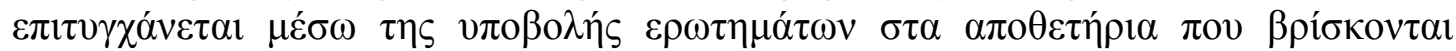

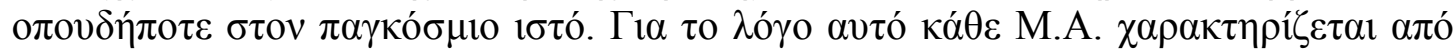

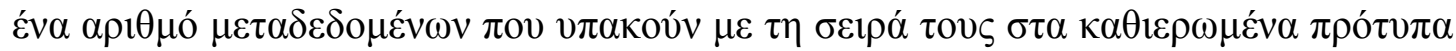
(ó $\pi \omega \varsigma \tau \alpha$ LOM standards $\tau\rceil \varsigma$ IEEE, $\eta$ $\tau$ ov Dublin core). $\Sigma \tau \eta \nu ~ \varepsilon \rho \gamma \alpha \sigma i ́ \alpha \tau \omega \nu$ Botsios $\kappa \alpha 1$

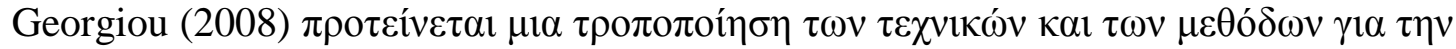

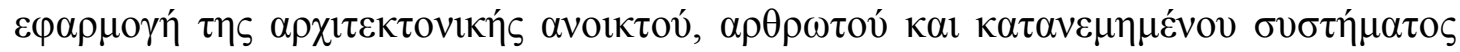

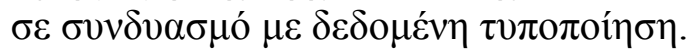

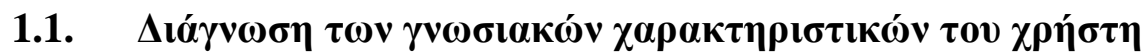

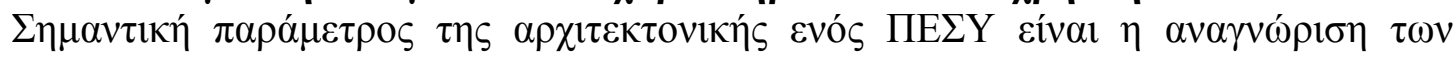

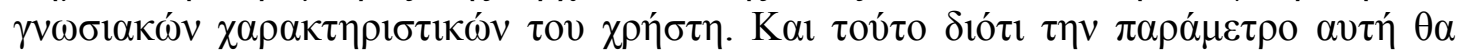

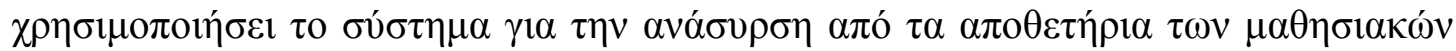

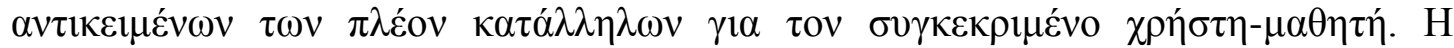

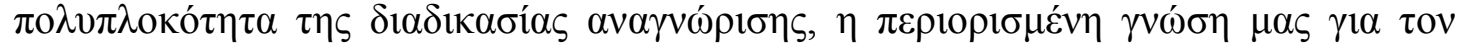

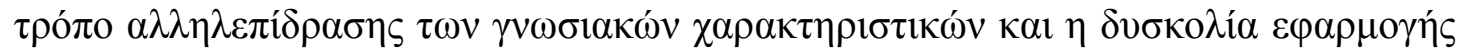

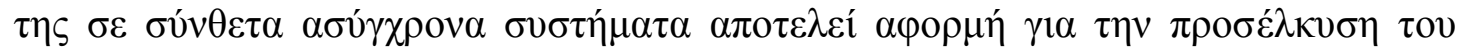

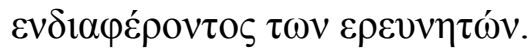

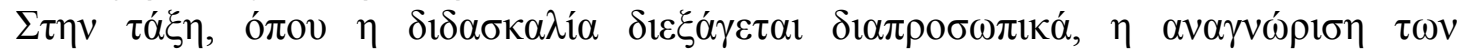

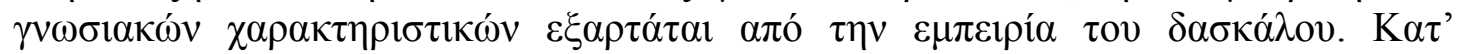

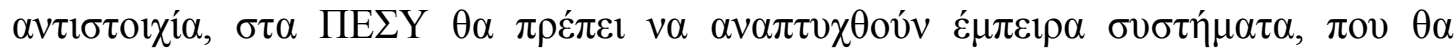

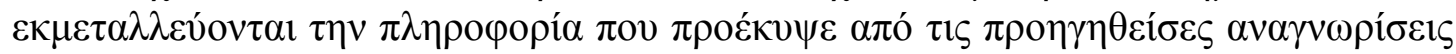

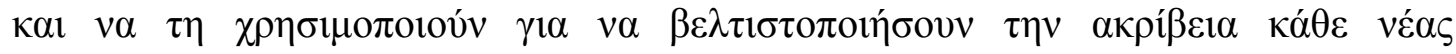
$\alpha v \alpha \gamma v \omega ́ \rho ı\rceil \eta s$.

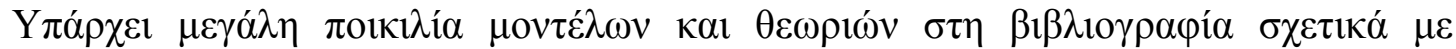

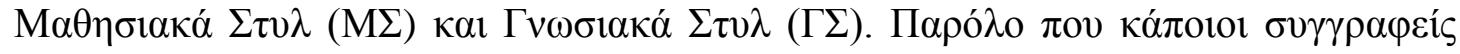




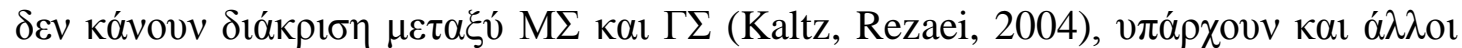

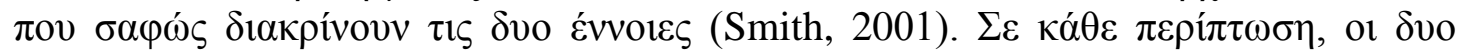

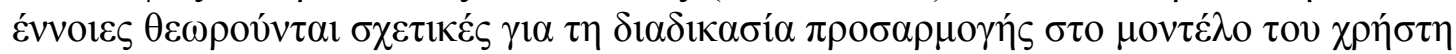

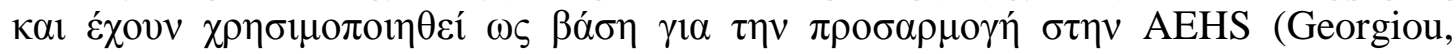

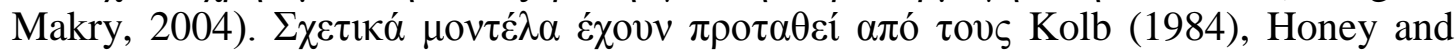
Mumford, Dunn R. and Dunn K. (1985 \& 1992), Felder and Silverman (1988),

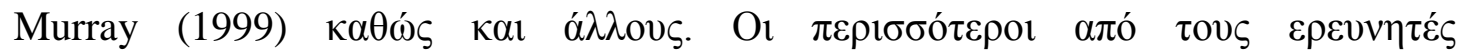

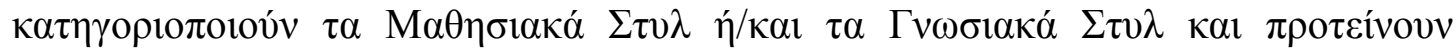

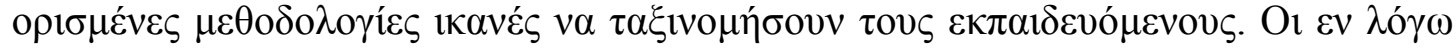

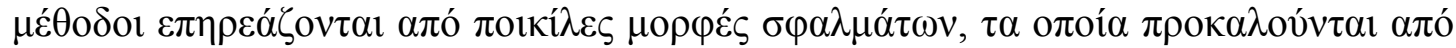

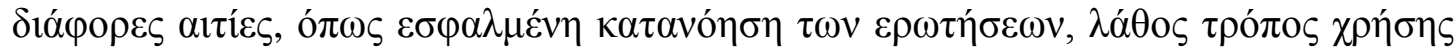

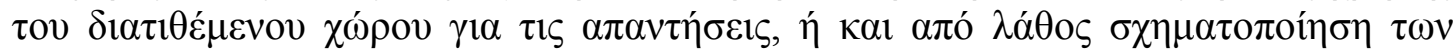

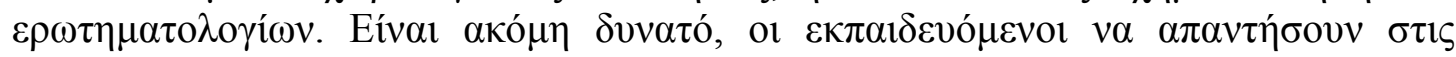

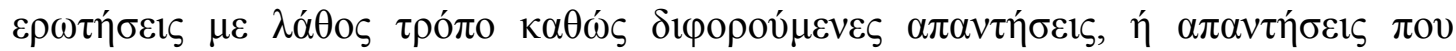

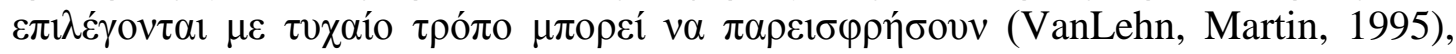

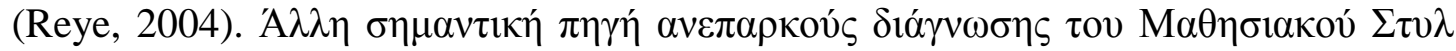

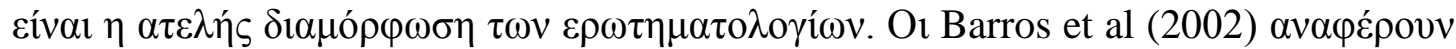

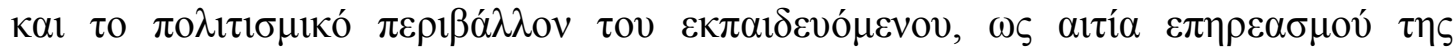

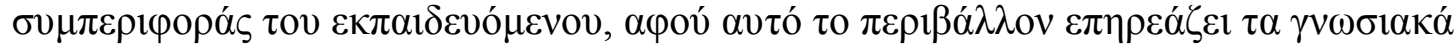

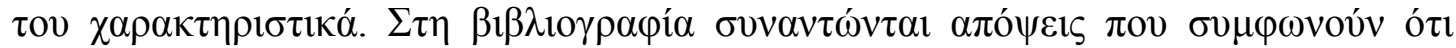

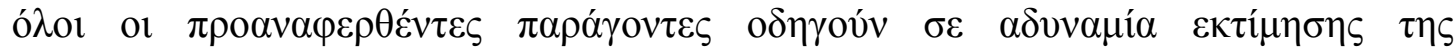

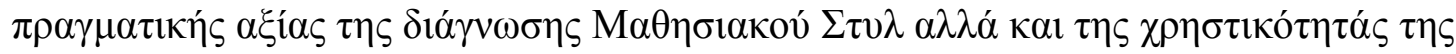

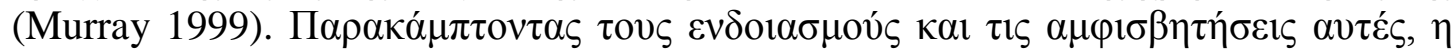

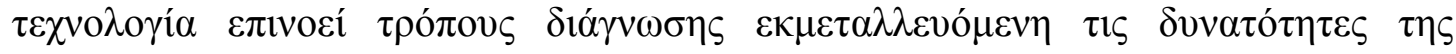

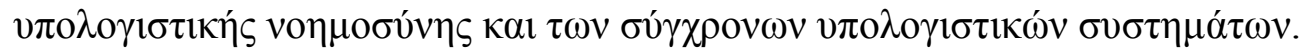

\section{2. $\Sigma \tau 0 \chi \alpha \sigma \tau \iota \kappa \alpha ́ ~ \varepsilon ́ \mu \pi \varepsilon เ \rho \alpha ~ \sigma v \sigma \tau \eta ́ \eta \alpha \tau \alpha^{5}$}

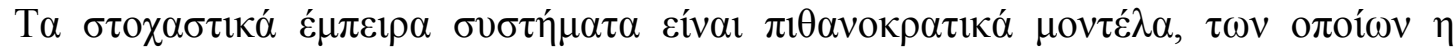

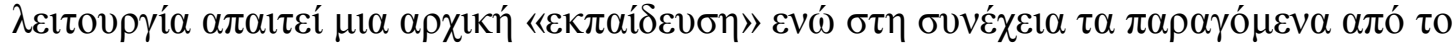

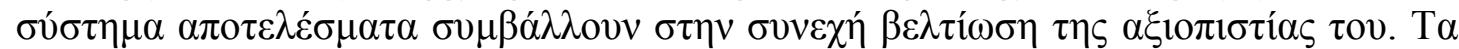

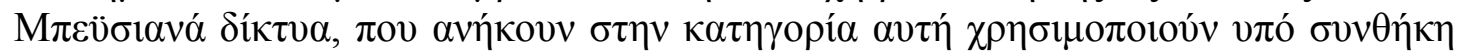

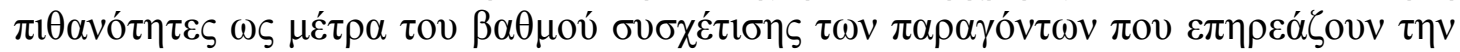

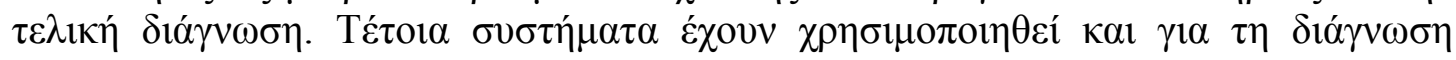

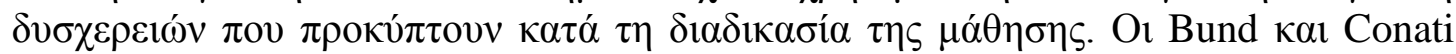

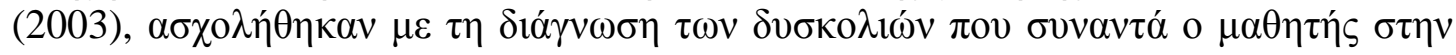

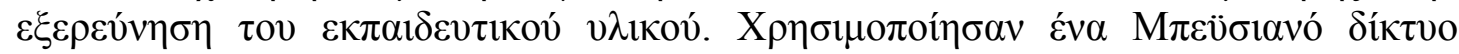

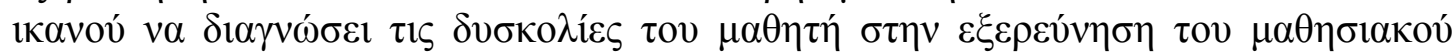

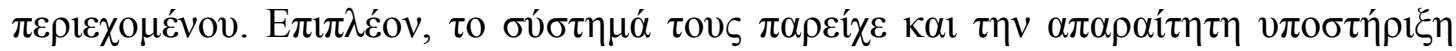

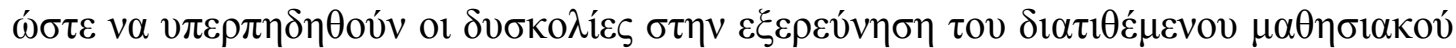

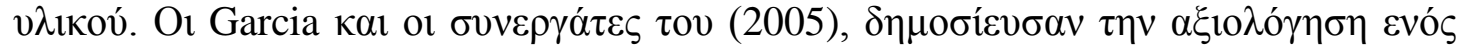

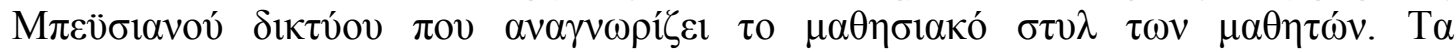

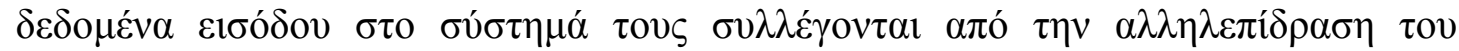

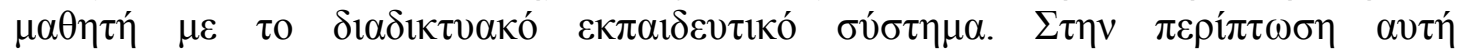

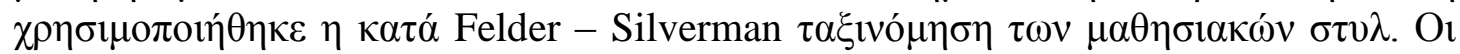

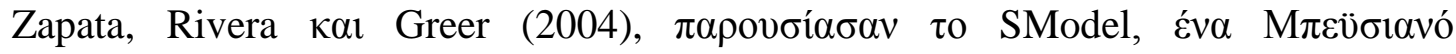

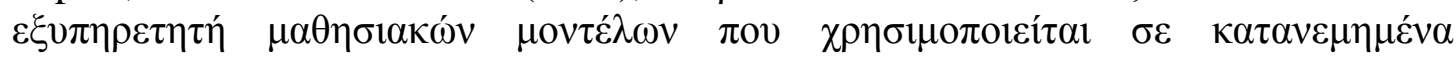

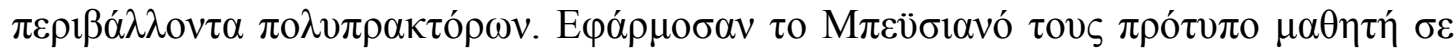

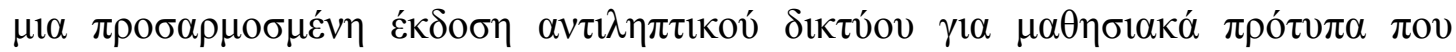

\footnotetext{
${ }^{5}$ Stochastic Expert Systems
} 


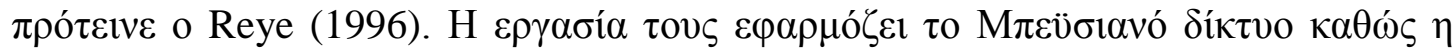

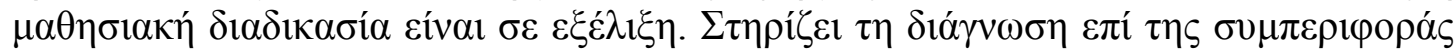

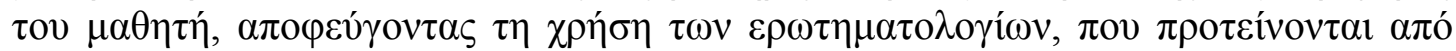

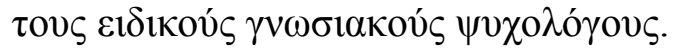

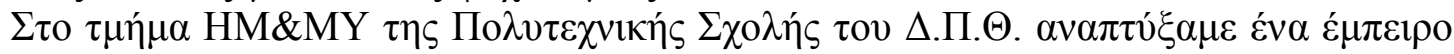

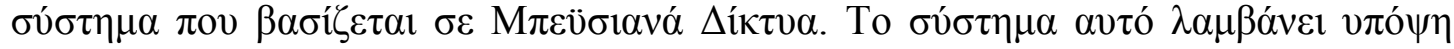

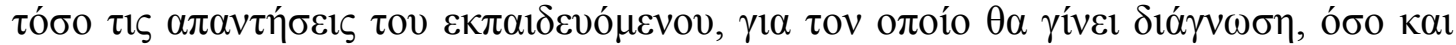

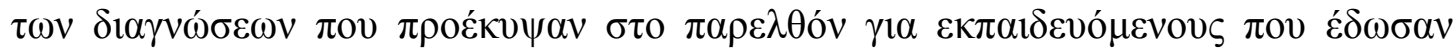

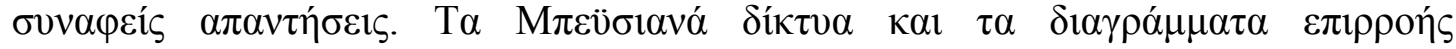

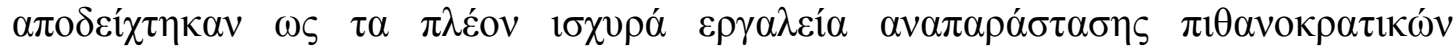

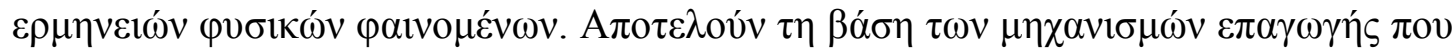

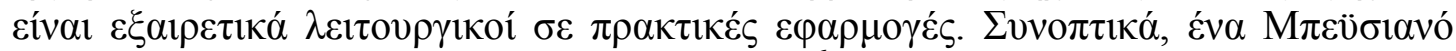

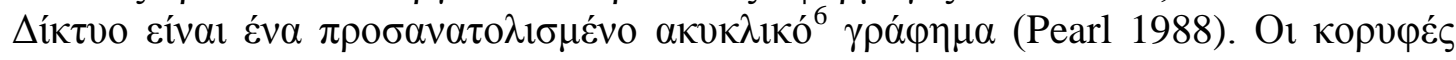

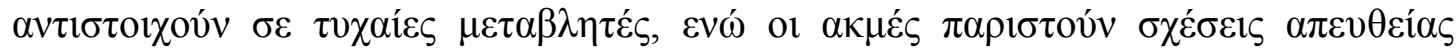

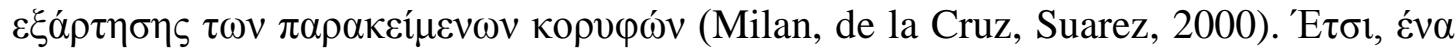

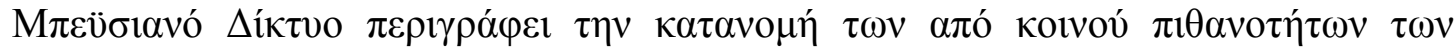

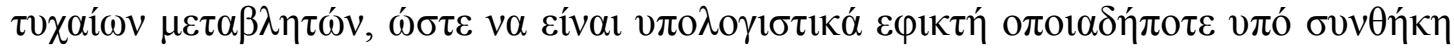

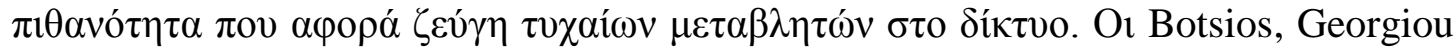

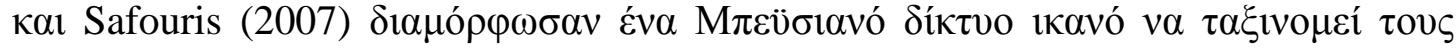

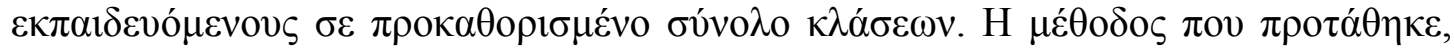

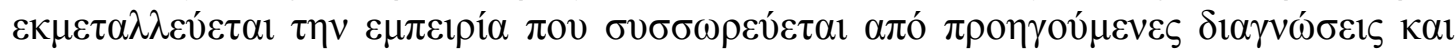

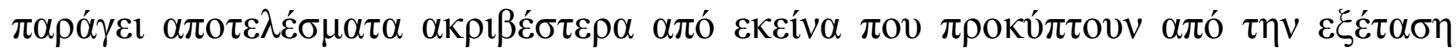

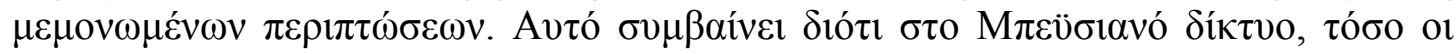

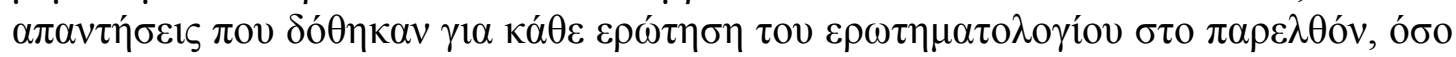

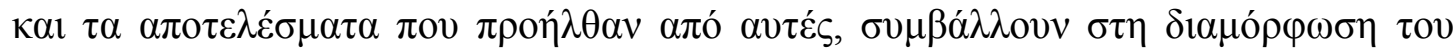

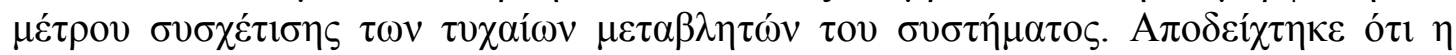

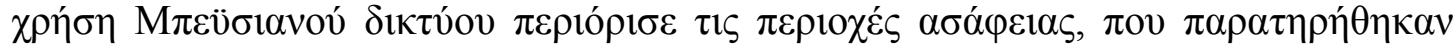

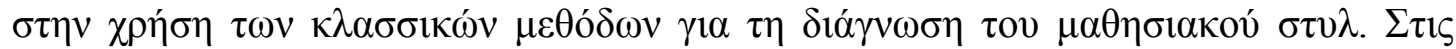

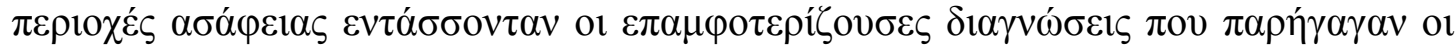

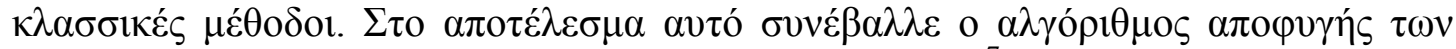

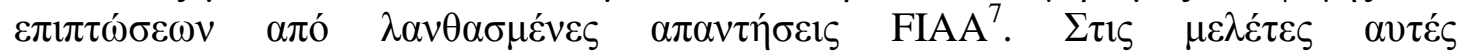

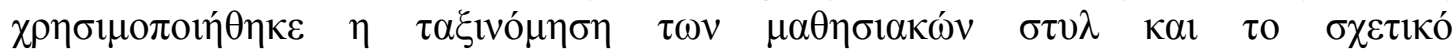

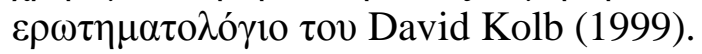

\subsection{Xрฑ์}

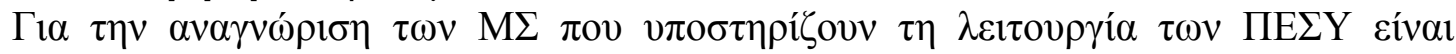

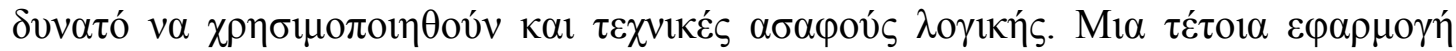

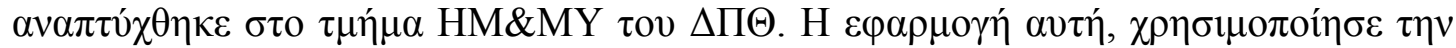

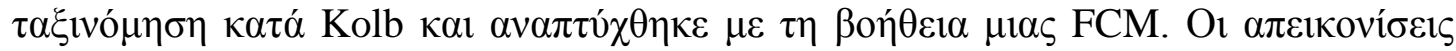

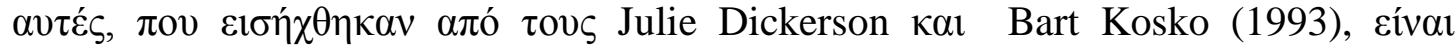

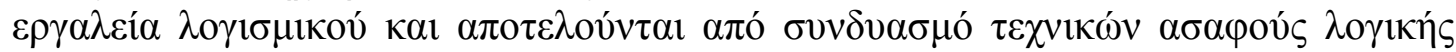

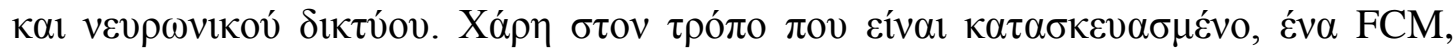

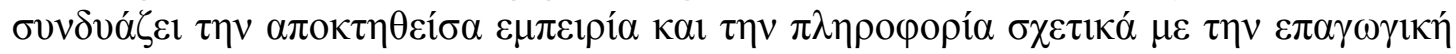

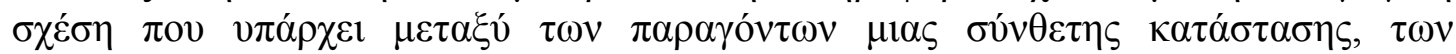

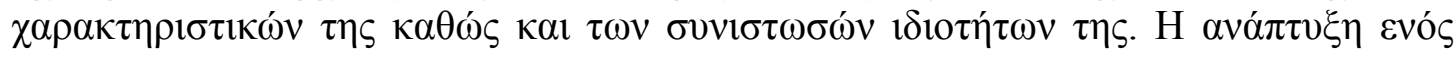

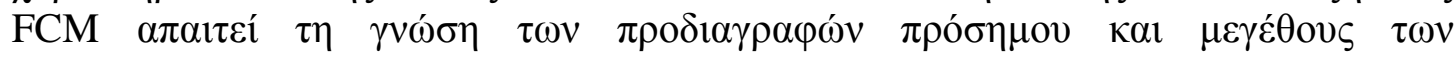

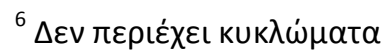

${ }^{7}$ Fault Implication Avoidance Algorithm (FIAA)

${ }^{8}$ Fuzzy Cognitive Map (FCM)
} 


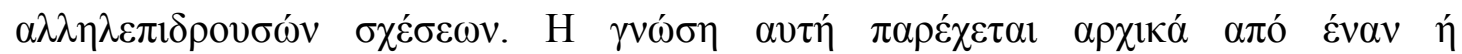

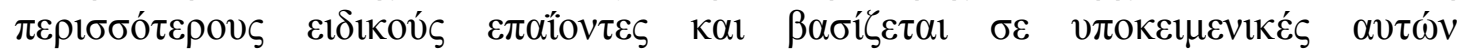

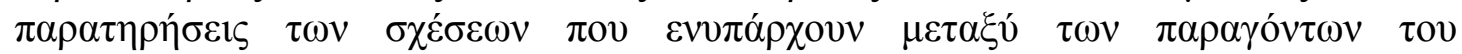

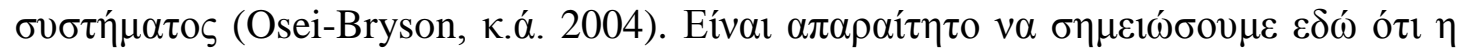

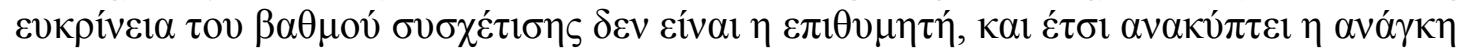

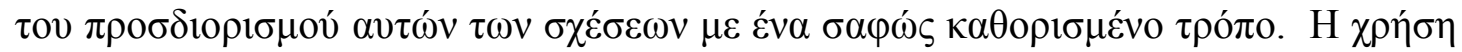

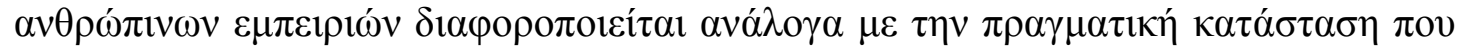
$\alpha \pi \varepsilon 1 \kappa o v i ́ \zeta \varepsilon \tau \alpha \imath$ (Georgopoulos, Malandraki, and Stylios, 2003).

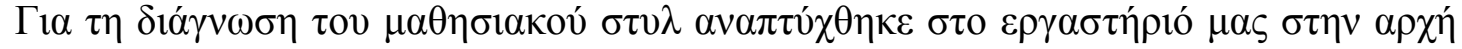

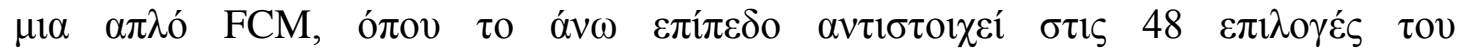

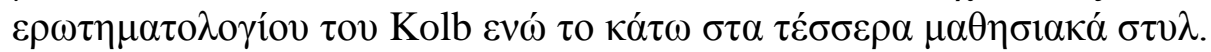

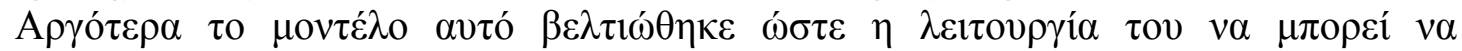

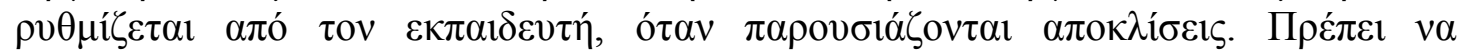

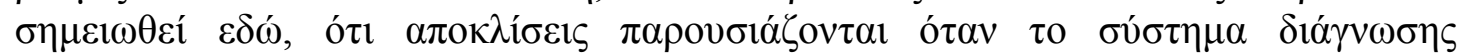

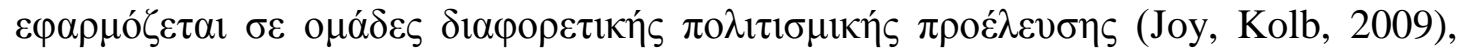

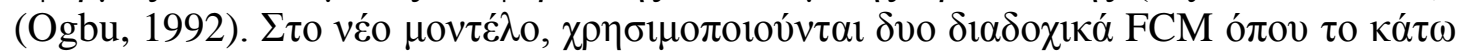

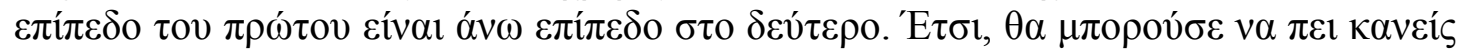

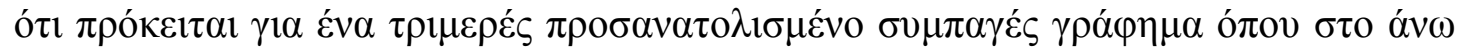

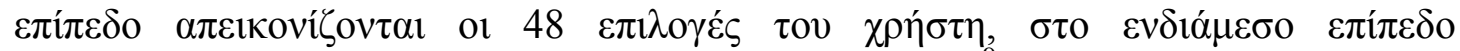

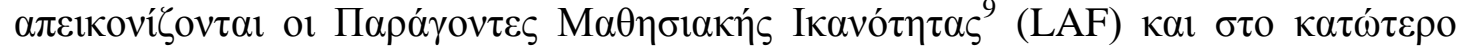

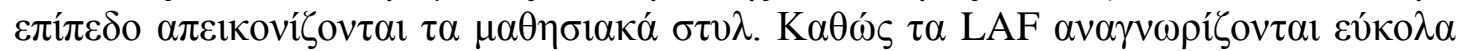

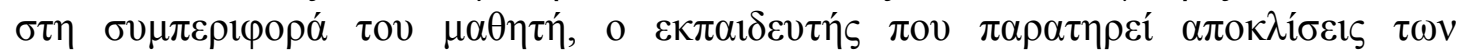

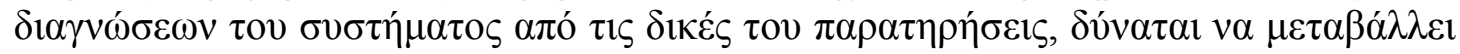

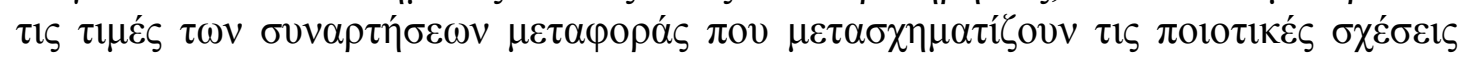

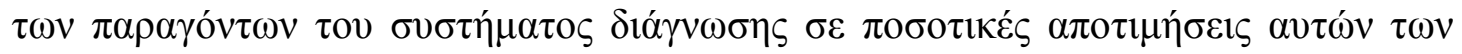

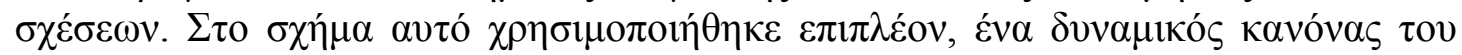

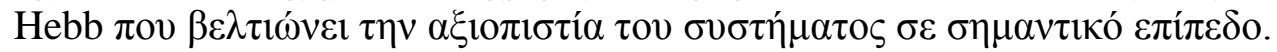

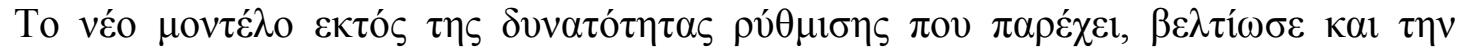

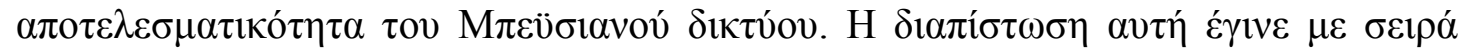

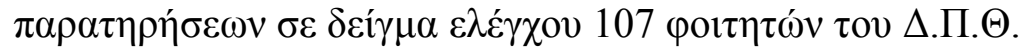

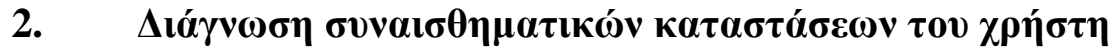

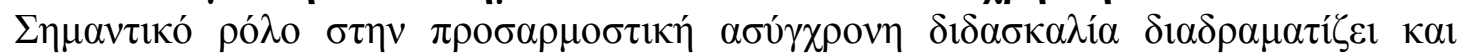

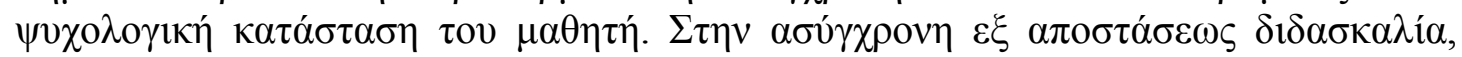

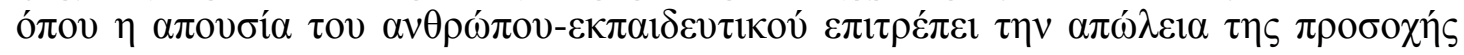

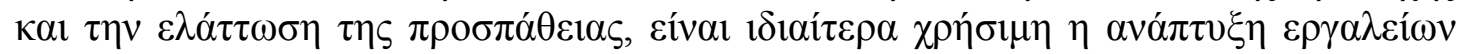

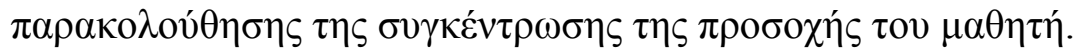

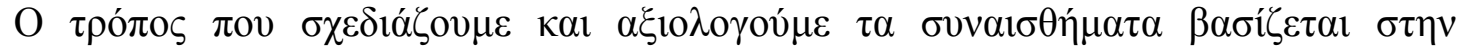

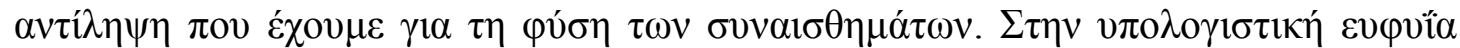

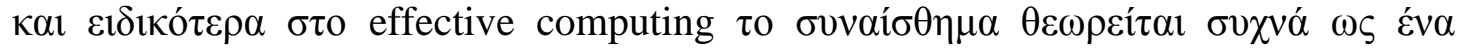

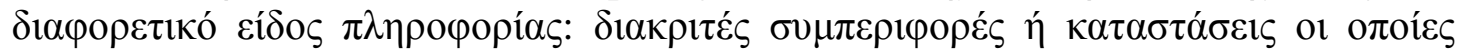

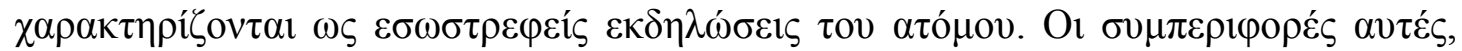

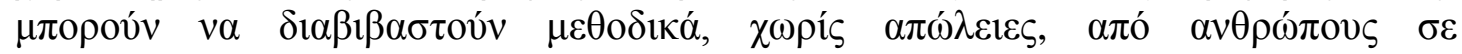

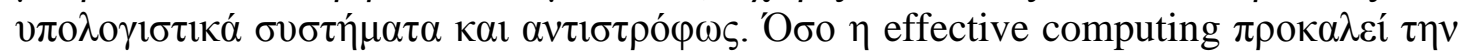

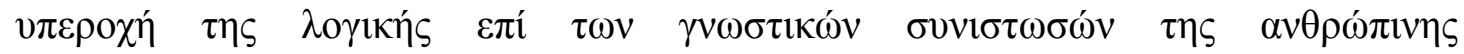

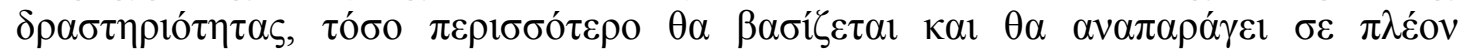

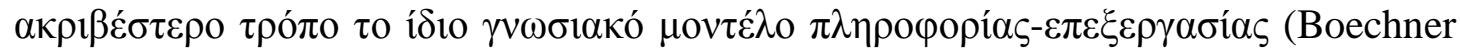

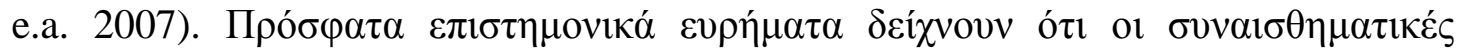

\footnotetext{
${ }^{9}$ Learning Ability Factors (LAF)
} 


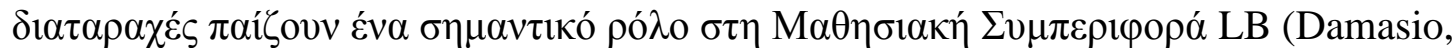

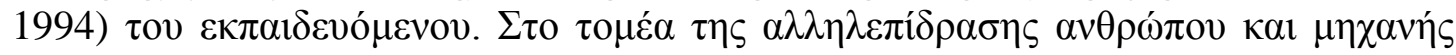

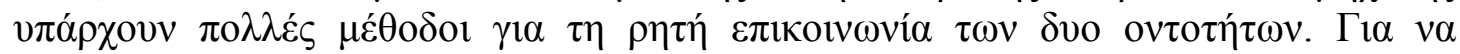

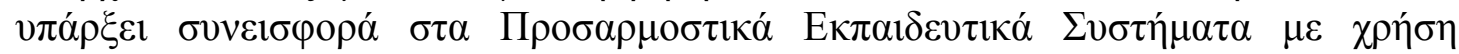

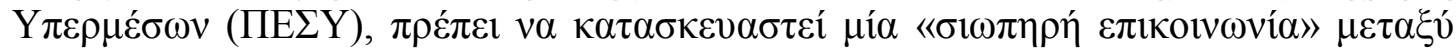

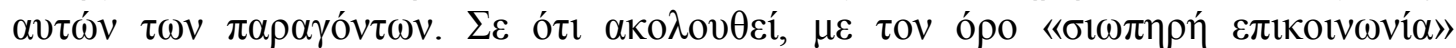

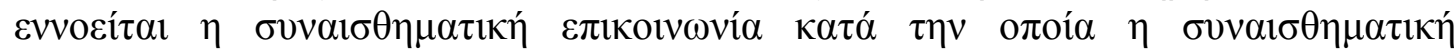

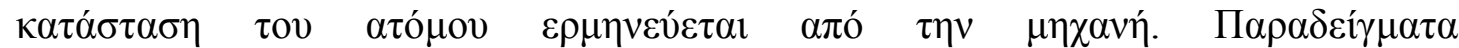

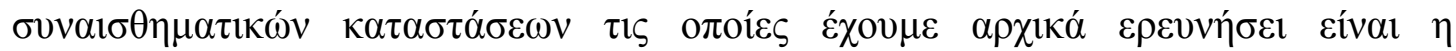

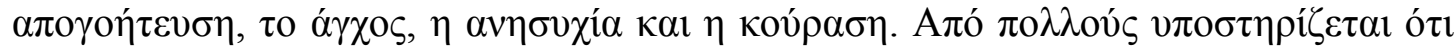

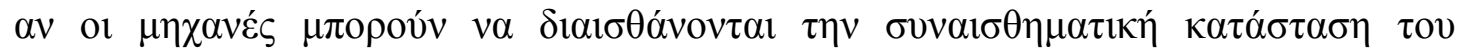

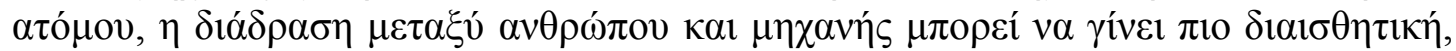

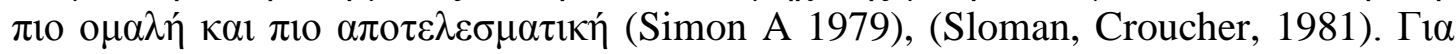

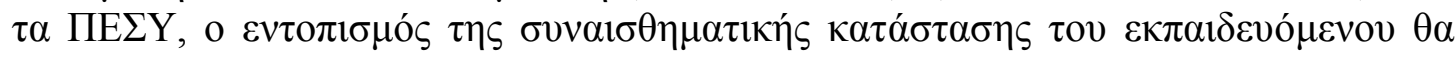

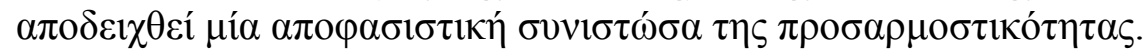

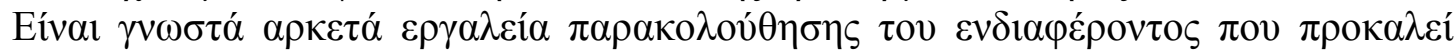

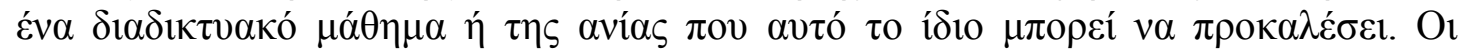

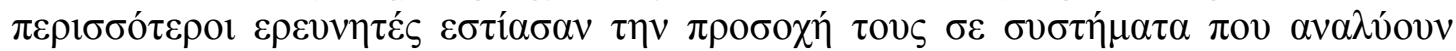

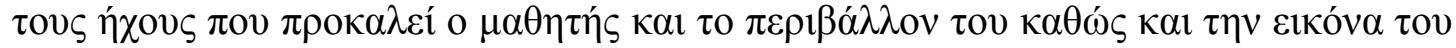
$\mu \alpha \theta \eta \tau \eta ் . ~ \Sigma \tau \eta ~ \sigma v v \varepsilon ́ \chi \varepsilon l \alpha ~ \tau \alpha ~ \delta \varepsilon \delta o \mu \varepsilon ́ v \alpha ~ \alpha v \alpha \lambda v ́ o v \tau \alpha l ~ \kappa \alpha l ~ \alpha v \alpha \gamma v \omega \rho i \zeta \zeta o v \tau \alpha l ~ \sigma v v \alpha l \sigma \theta \eta \mu \alpha \tau 1 \kappa \varepsilon ́ \varsigma$

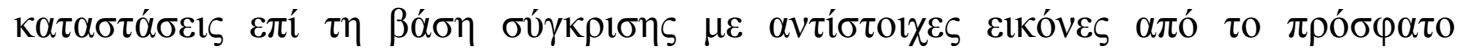
$\pi \alpha \rho \varepsilon \lambda \theta$ óv $\tau$ ov $\mu \alpha \theta \eta \tau \eta ́$.

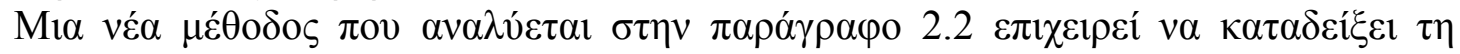

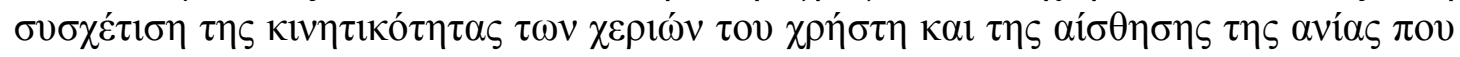

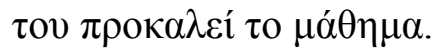

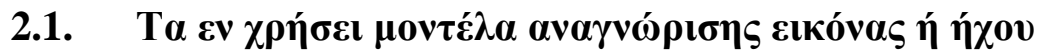

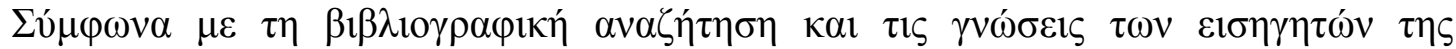

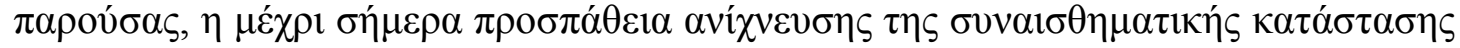

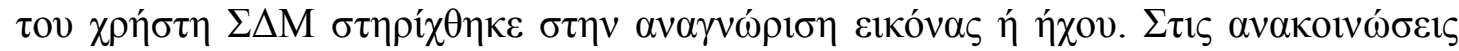

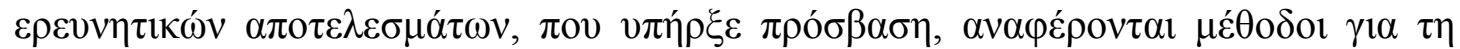

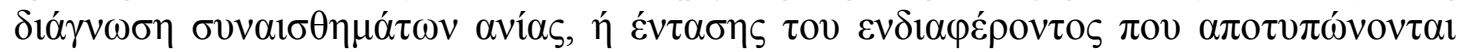

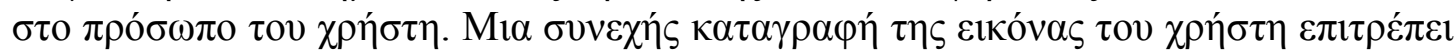

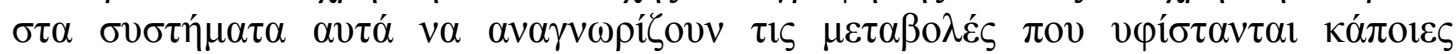

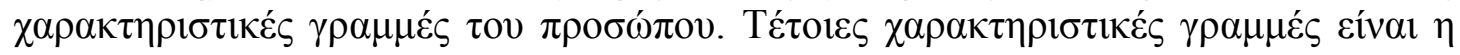

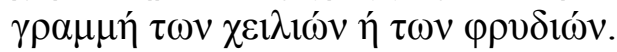

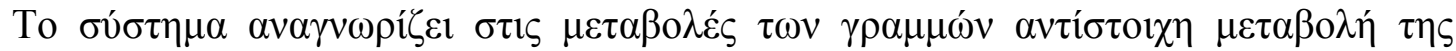

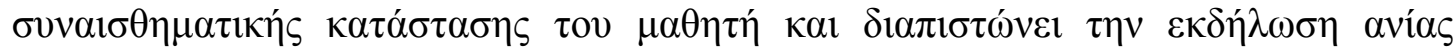

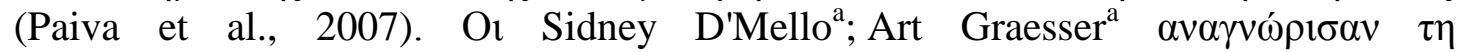

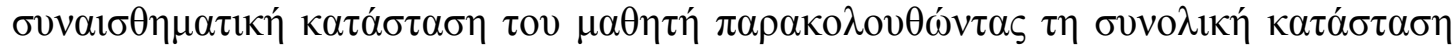

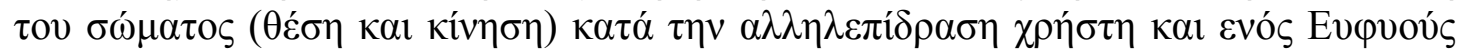

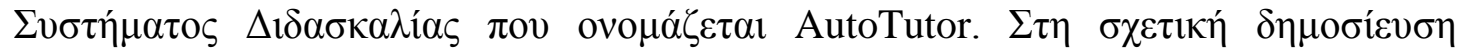

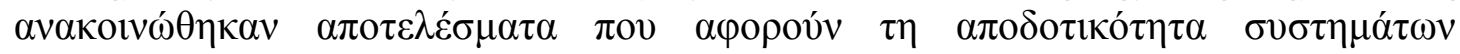

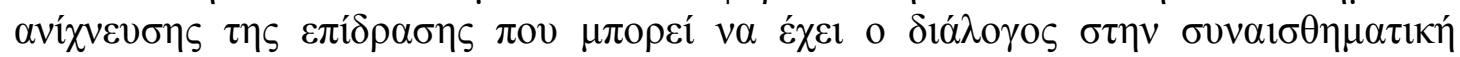

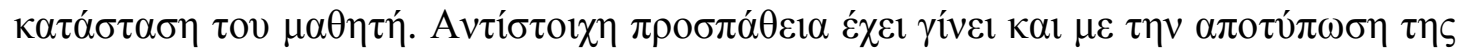

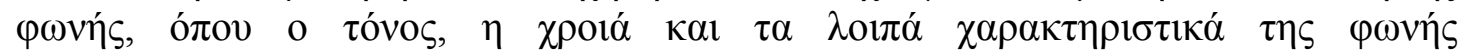

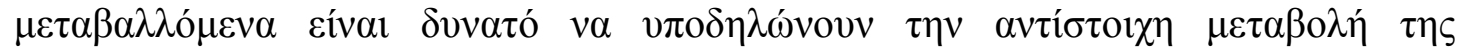

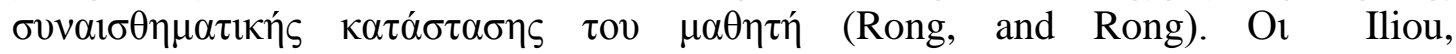

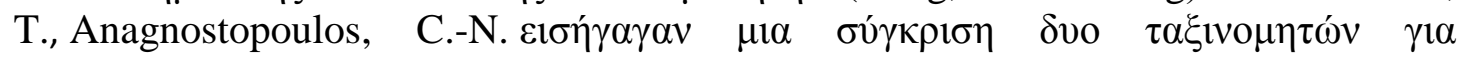

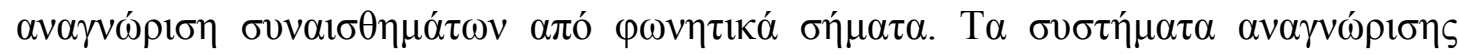




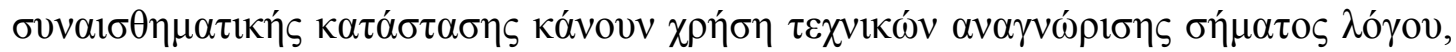

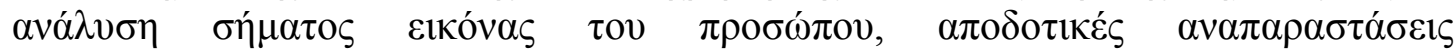

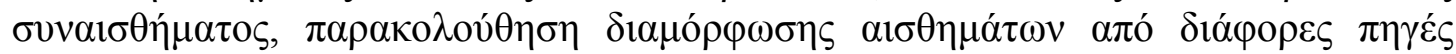

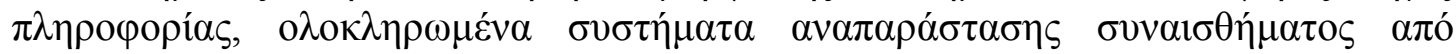

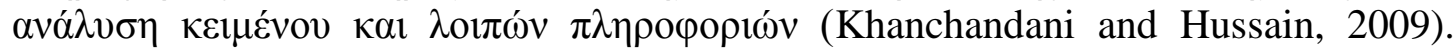

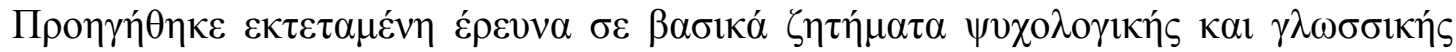

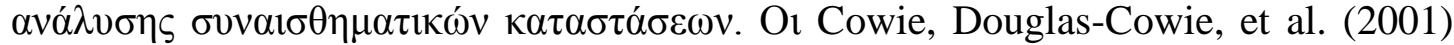

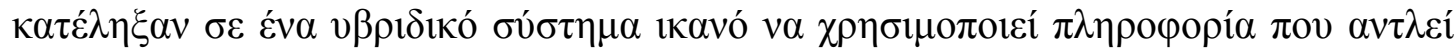

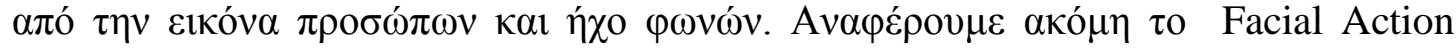

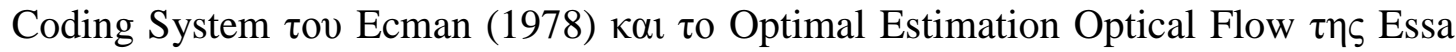
кaı Pentland (1997).

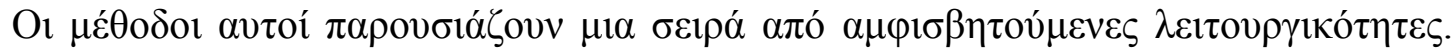

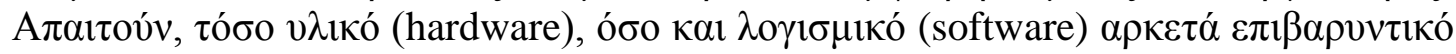

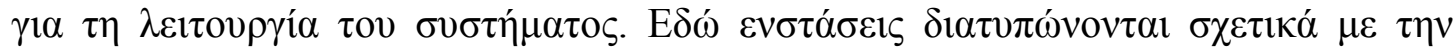

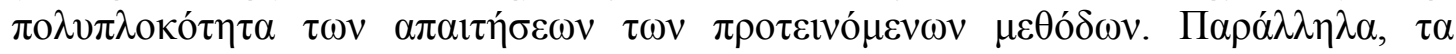

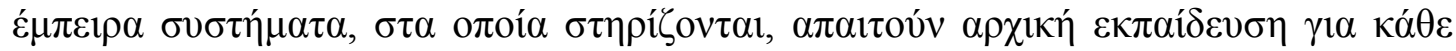

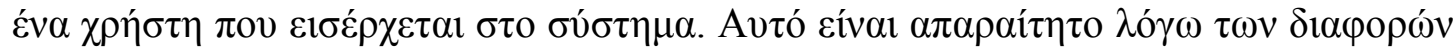

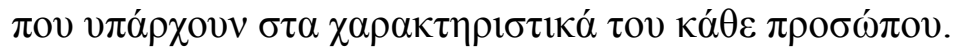

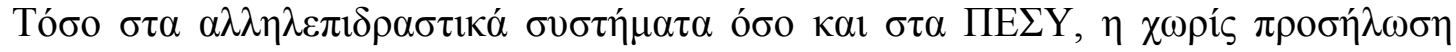

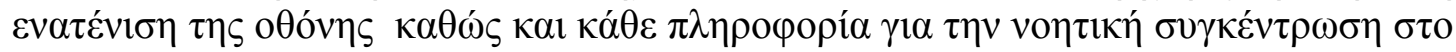

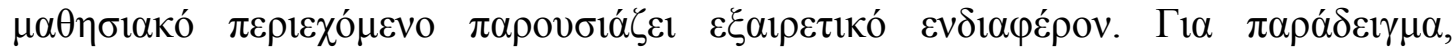

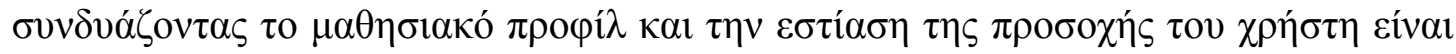

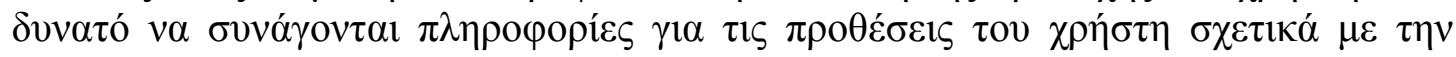

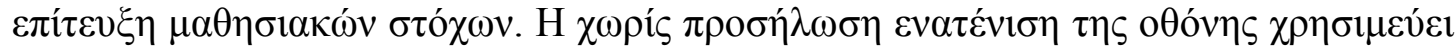

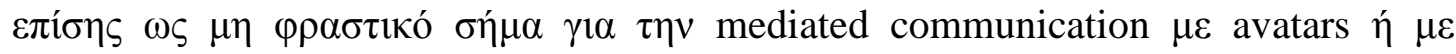

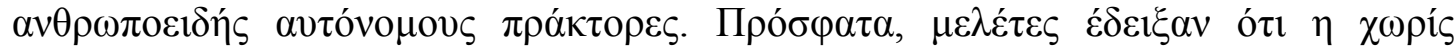

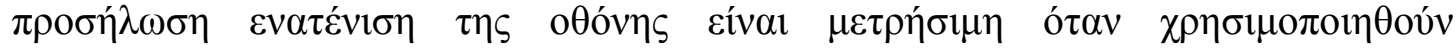

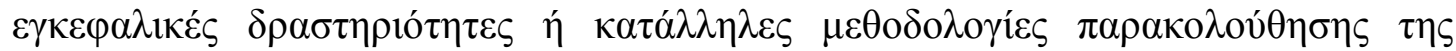

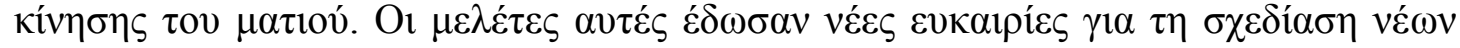

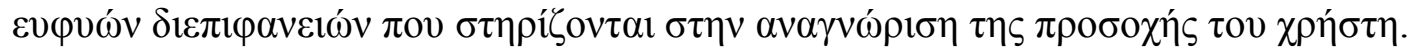

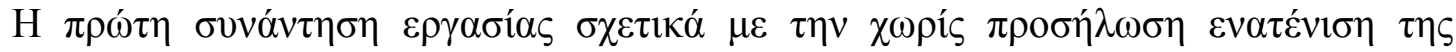

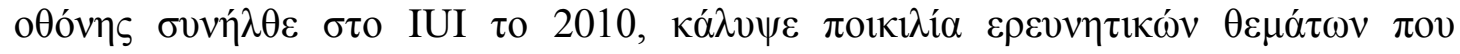

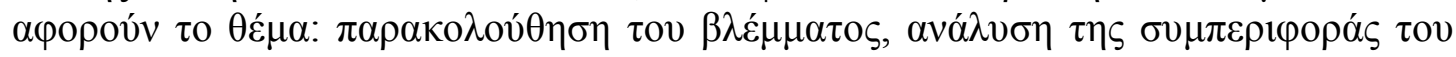

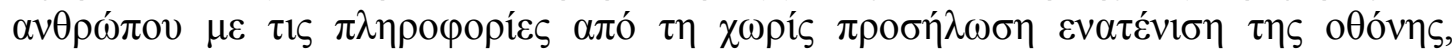

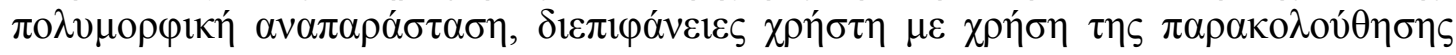

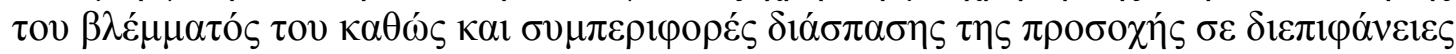

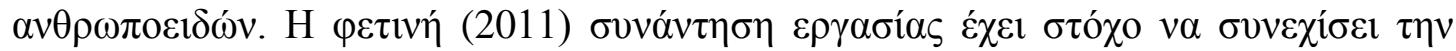

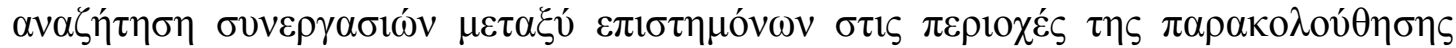

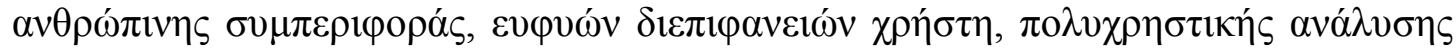

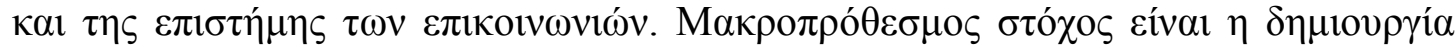

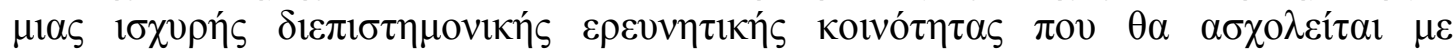

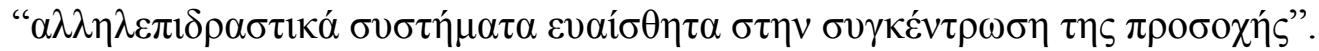

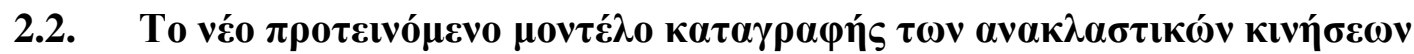

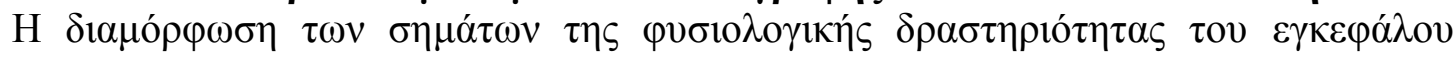

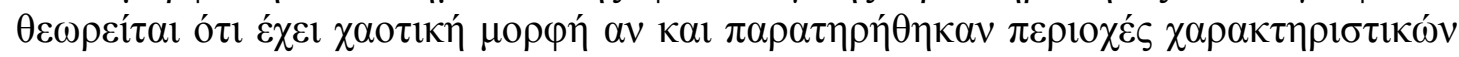

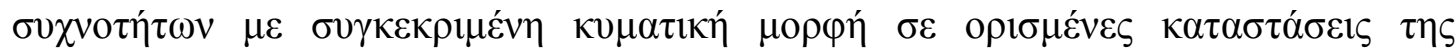

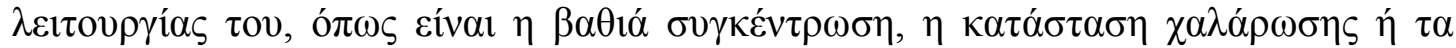

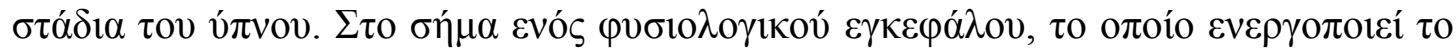

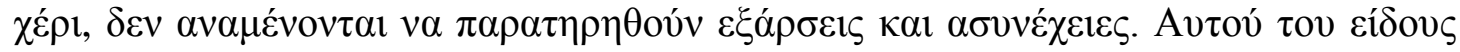




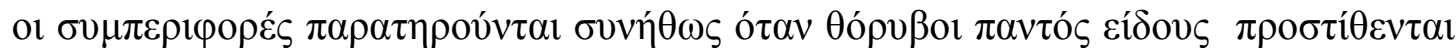

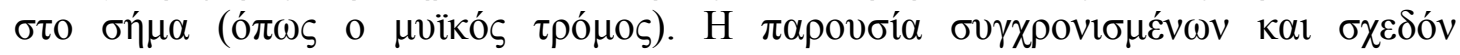

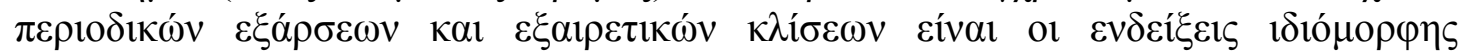

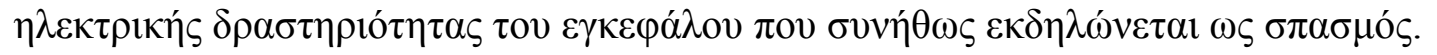

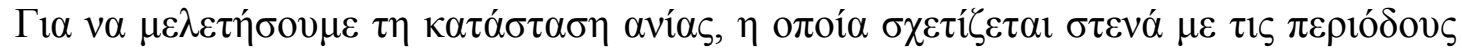

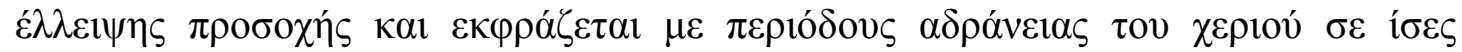

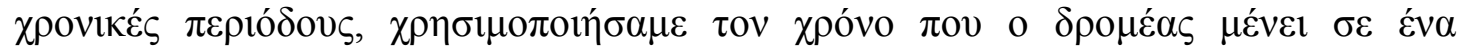

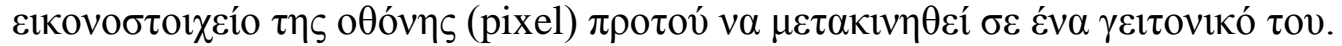

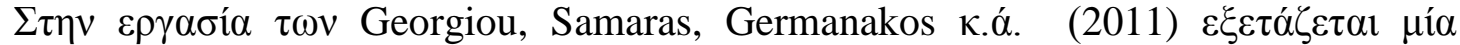

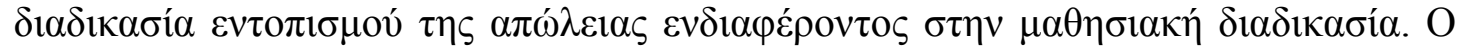

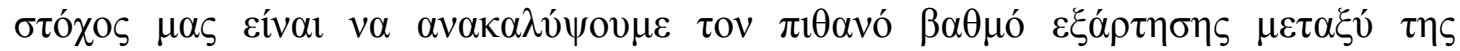

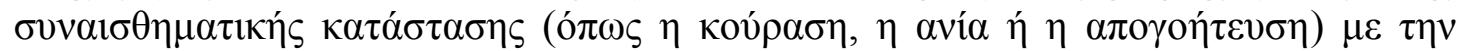

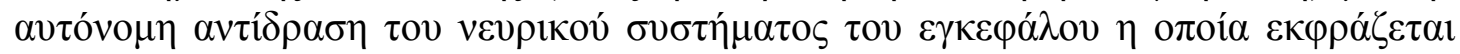

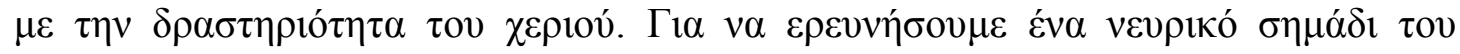

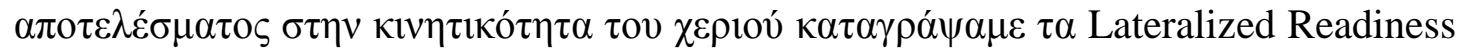

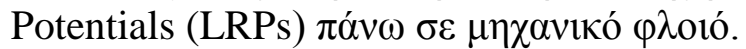

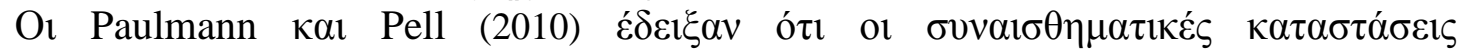

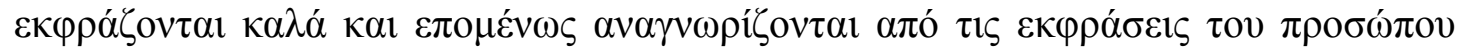

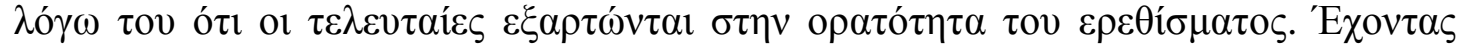

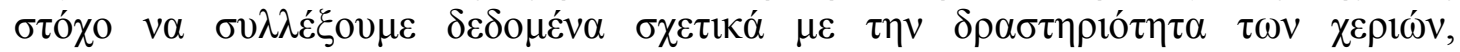

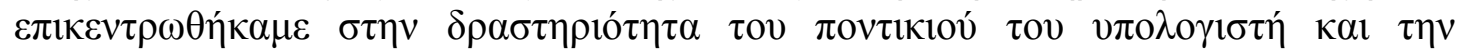

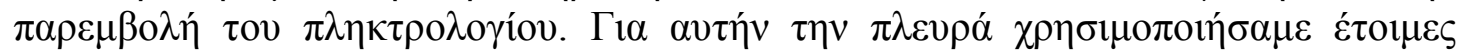

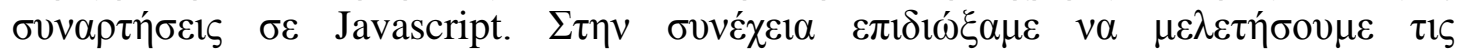

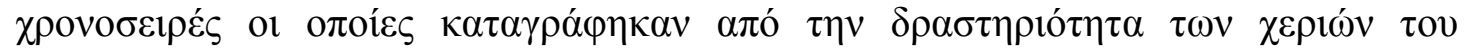

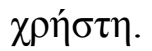

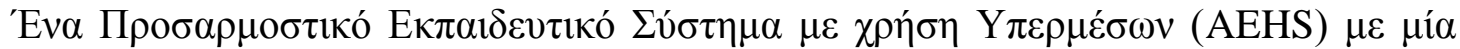

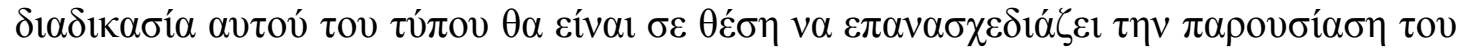

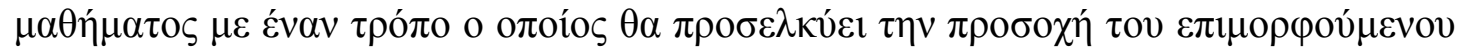

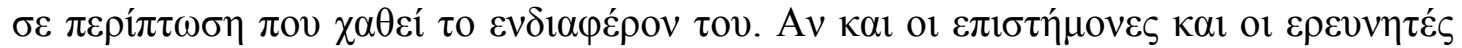

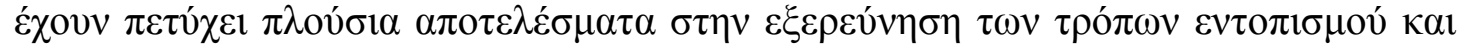

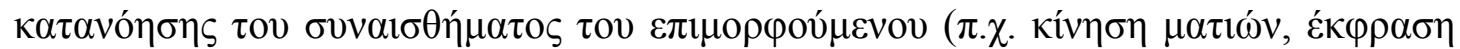

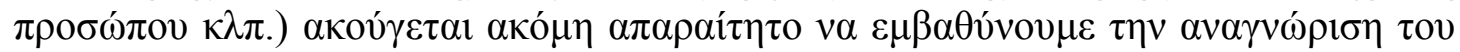

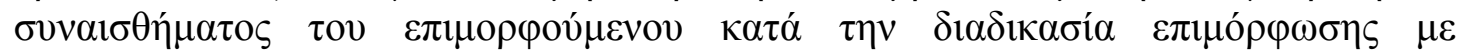

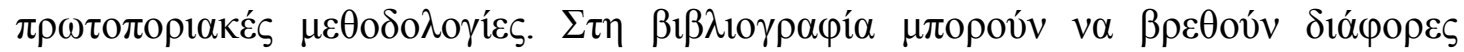

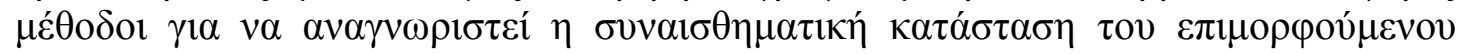

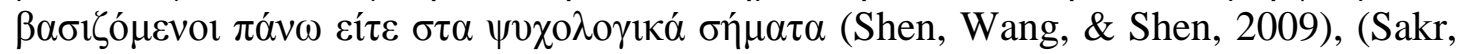

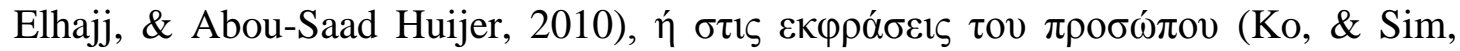

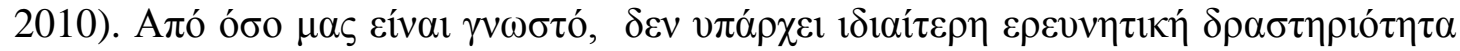

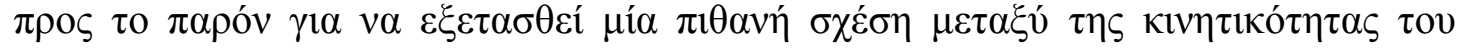

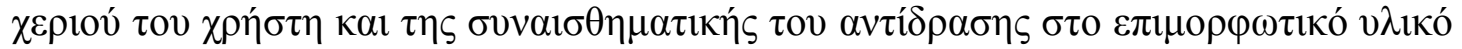

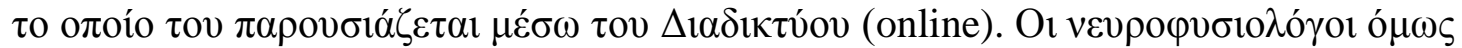

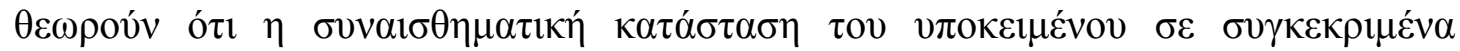

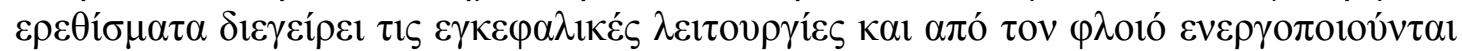

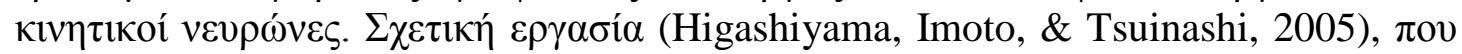

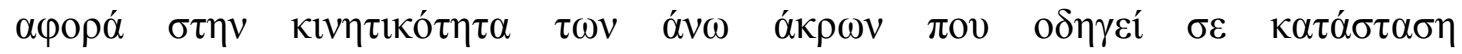

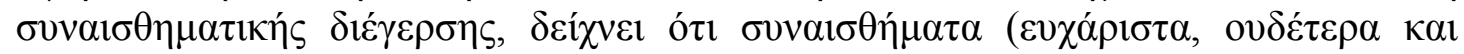

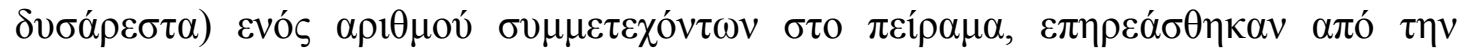

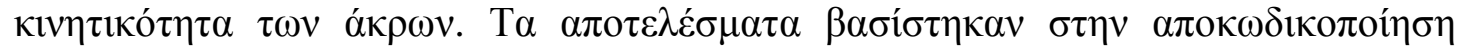

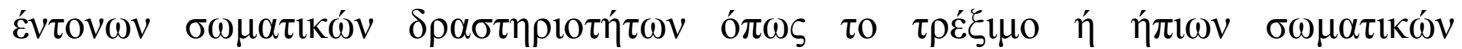

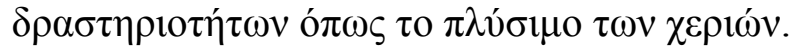




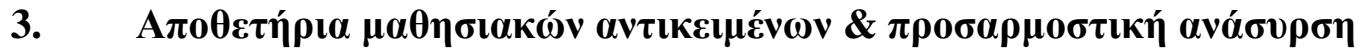

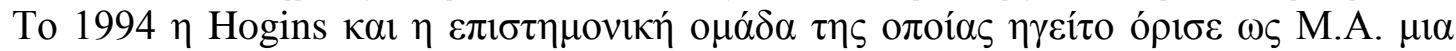

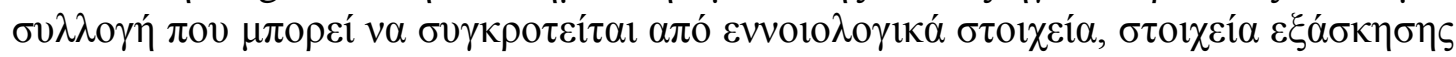

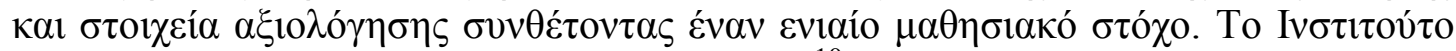

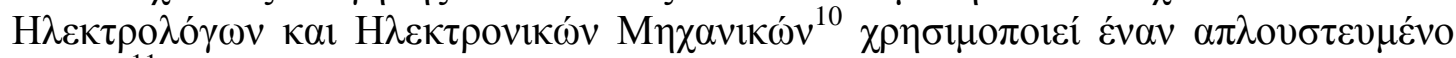

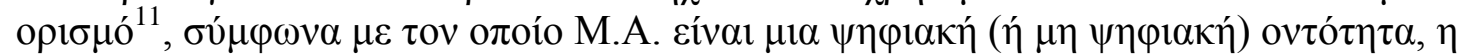

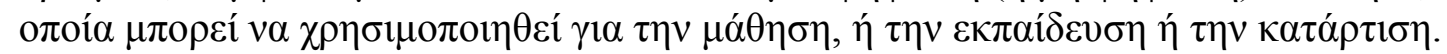

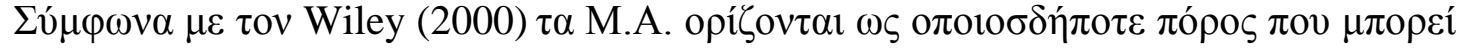

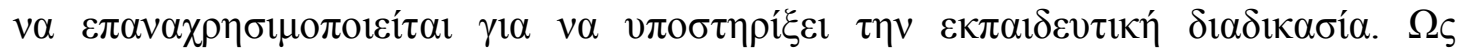

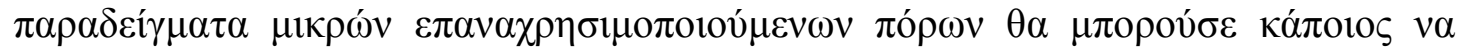

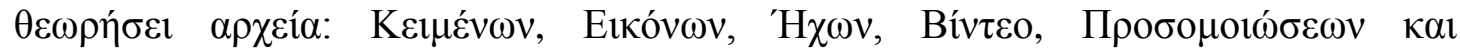

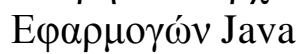

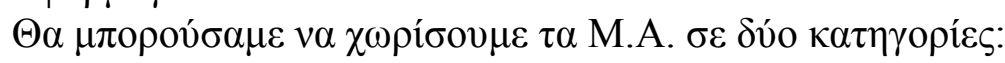

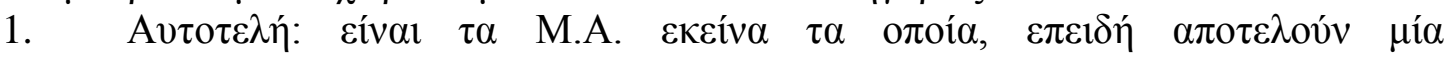

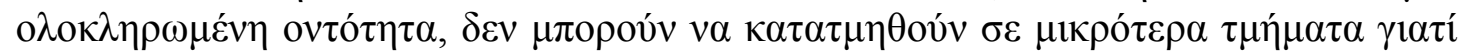

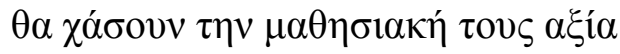

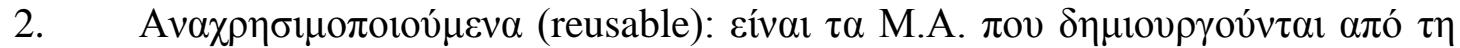

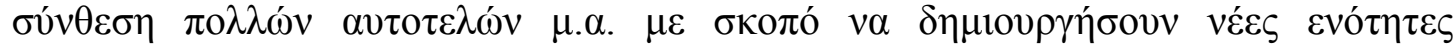

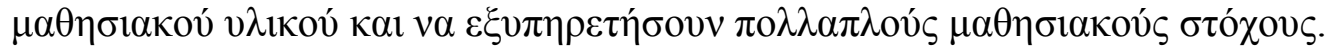

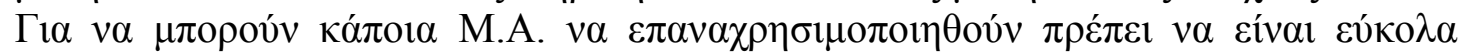

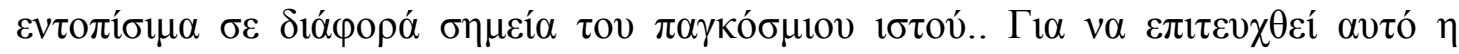

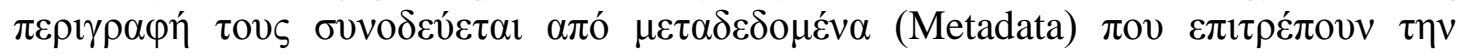

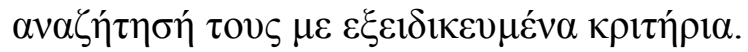

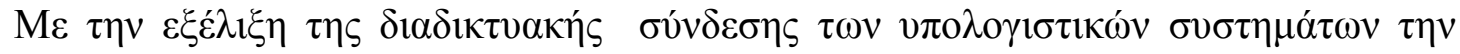

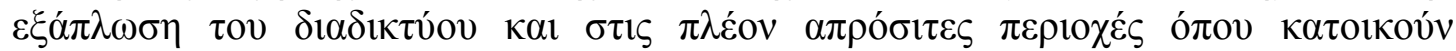

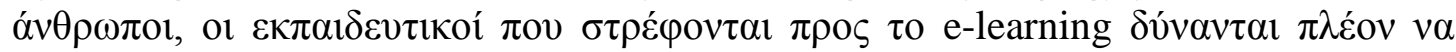

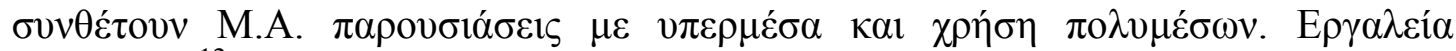

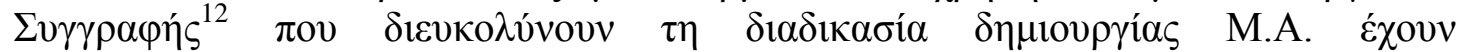

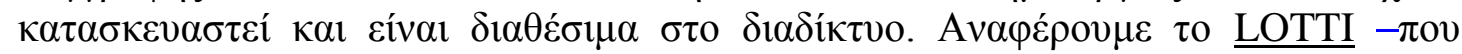

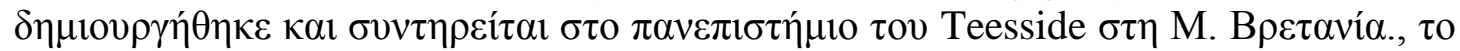

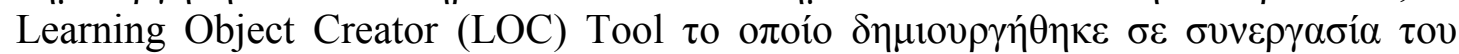

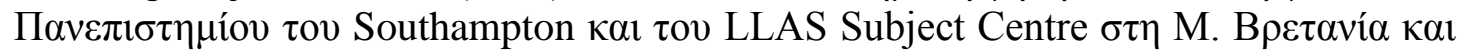

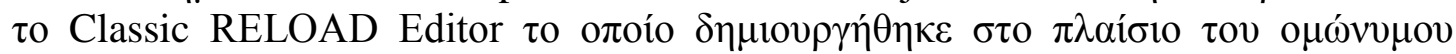
غ́pyov (RELOAD project)

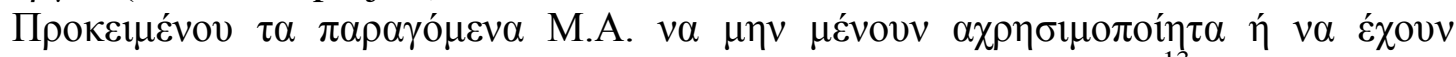

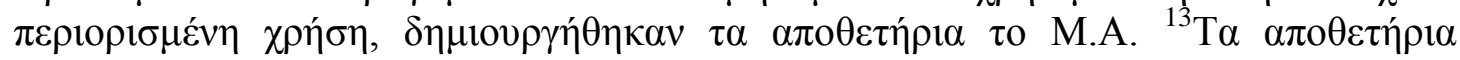

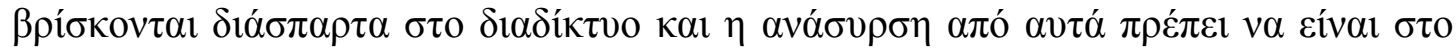

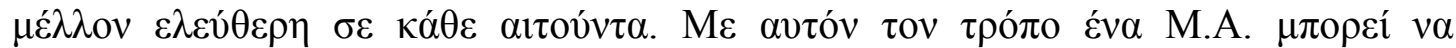

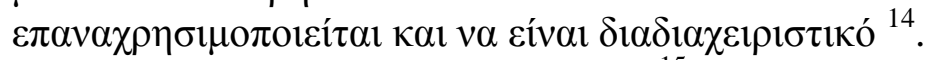

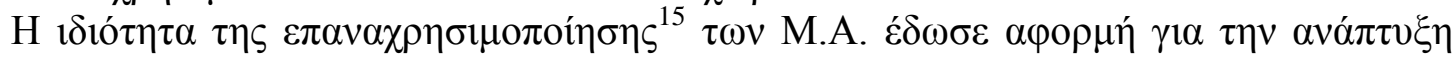

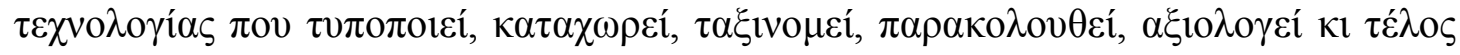

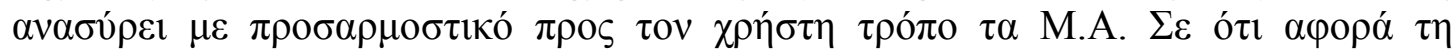

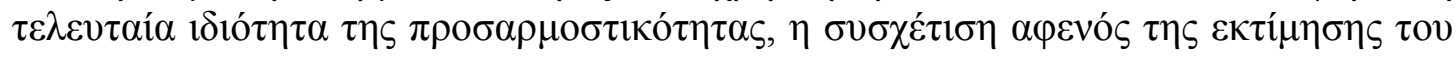

\footnotetext{
${ }^{10}$ Institute of Electrical and Electronic Engineers (IEEE)

${ }^{11}$ http://Itsc.ieee.org/wg12/files/LOM_1484_12_1_v1_Final_Draft.pdf

${ }^{12}$ Authoring Tool

${ }^{13}$ Learning Object Repositories

14 Interoperable

${ }^{15}$ Reusability
} 


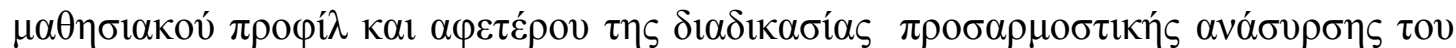

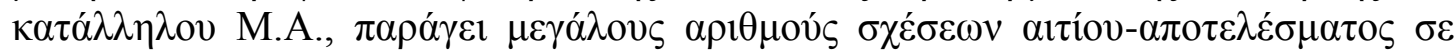

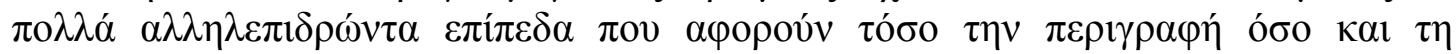

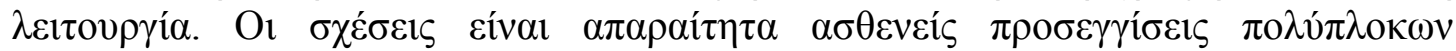

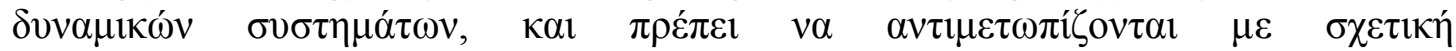

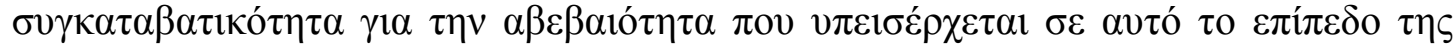

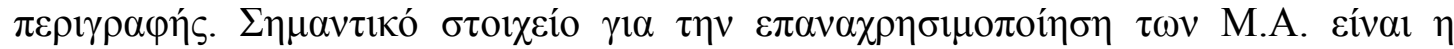

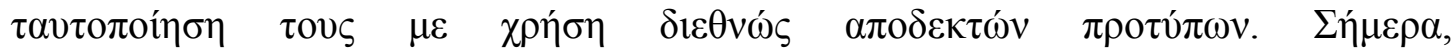

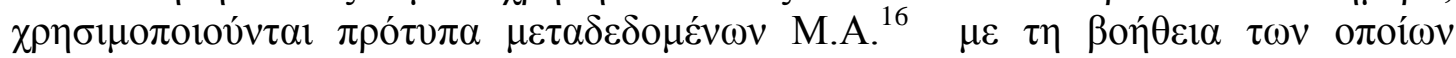
$\tau \alpha v \tau$ o

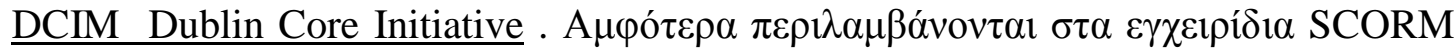

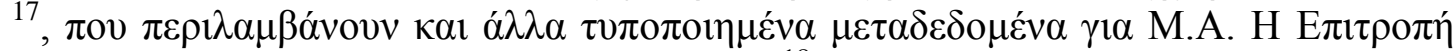

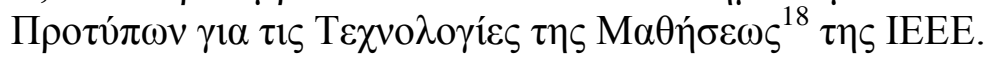

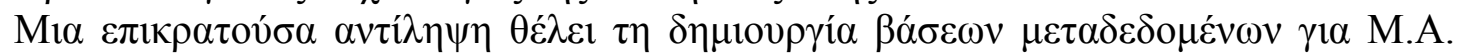

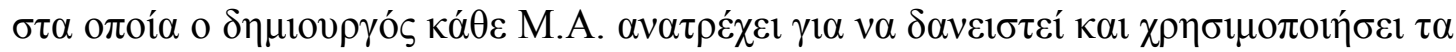

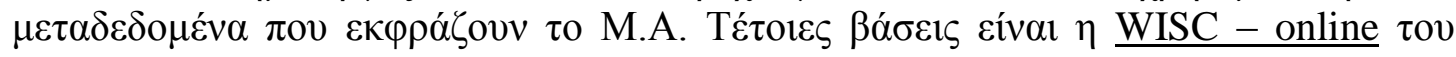
$\pi \alpha v \varepsilon \pi \imath \sigma \tau \eta \mu$ íov $\tau$ ov Wisconsin, $\tau$ o MERLOT -- Multimedia Educational Resource for Online Learning and Teaching, $\tau$ o North Carolina Learning Object Repository $\sigma \tau \iota \varsigma$

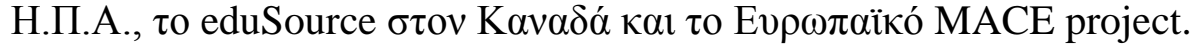

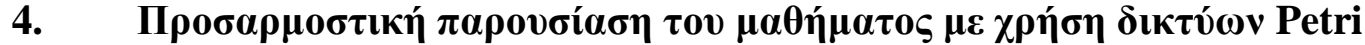

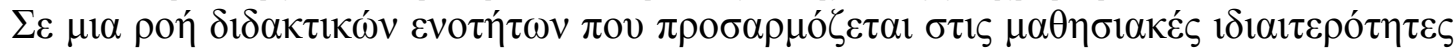

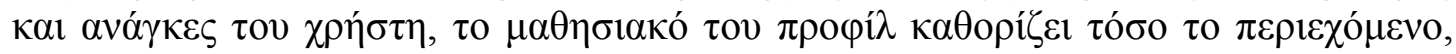

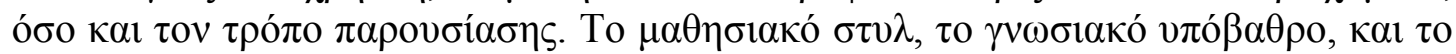

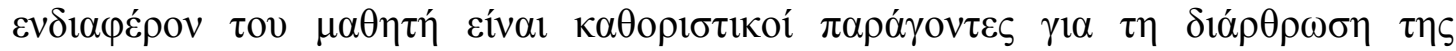

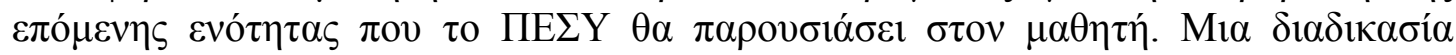

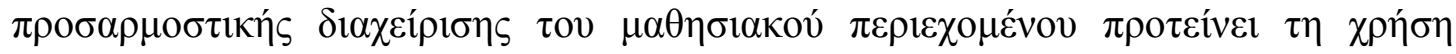

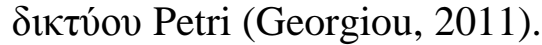

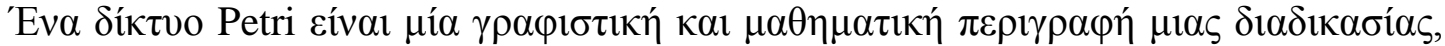

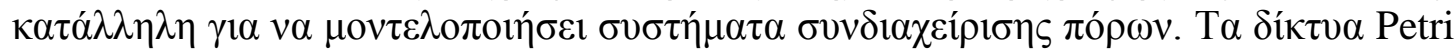

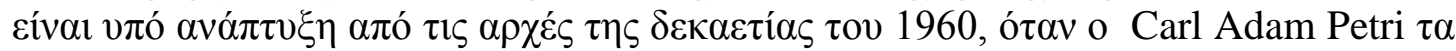

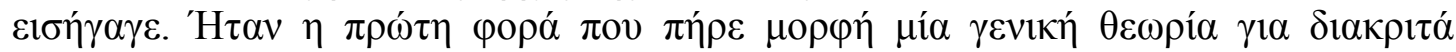

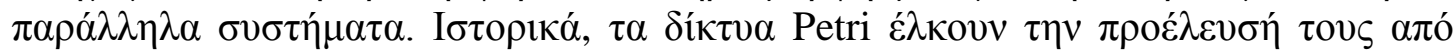

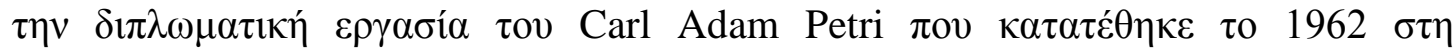

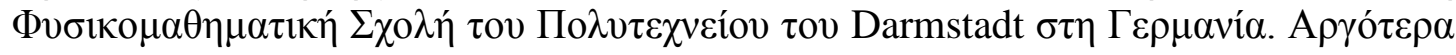

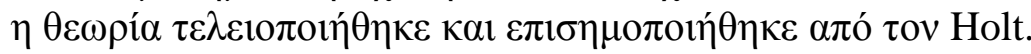

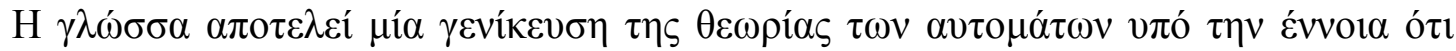

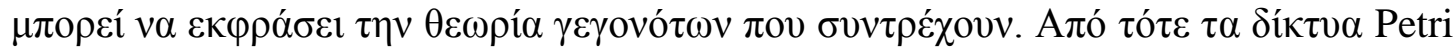

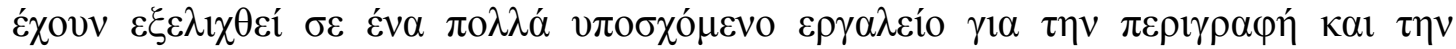

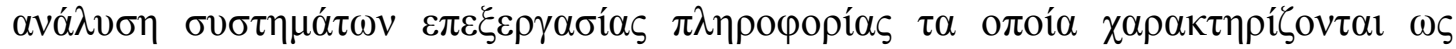

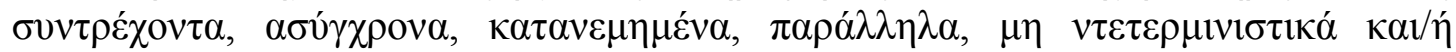

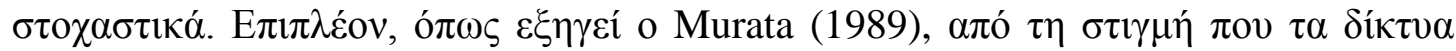

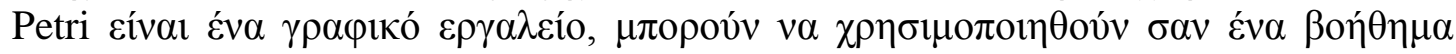

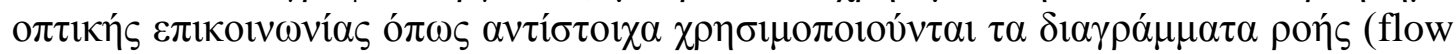

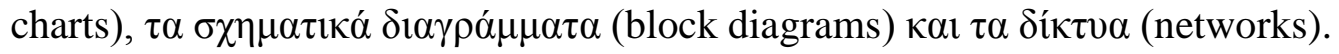

\footnotetext{
${ }^{16}$ Learning Object Metadata (LOM)

${ }^{17}$ Sharable Content Object Reference Model

${ }^{18}$ Learning Technologies Standard Committee
} 


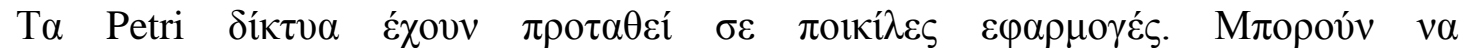

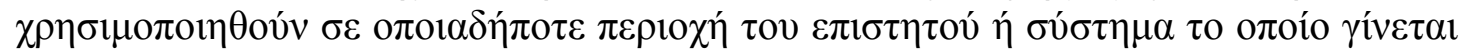

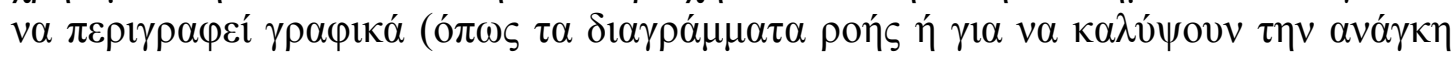

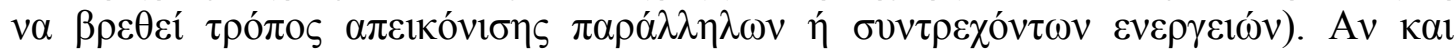

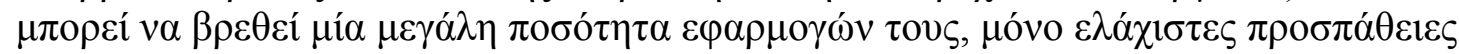

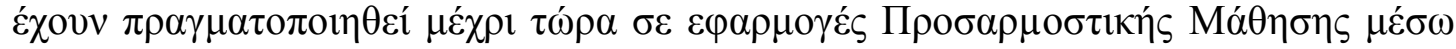

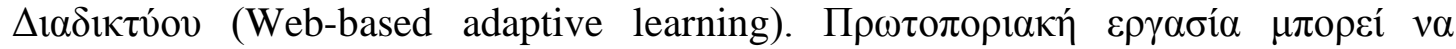

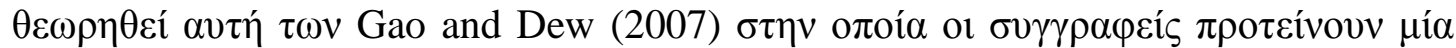

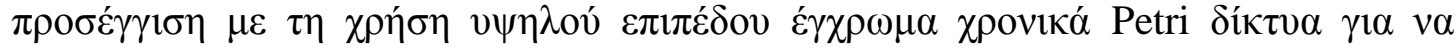

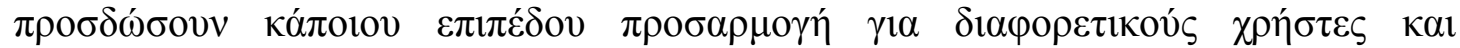

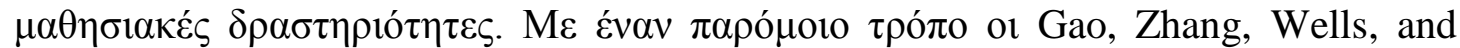

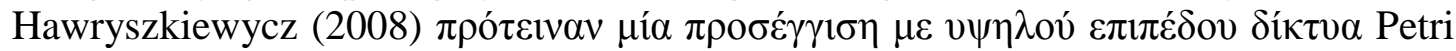

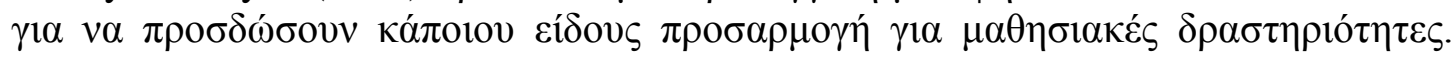

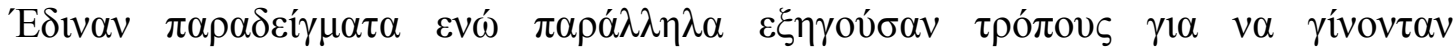

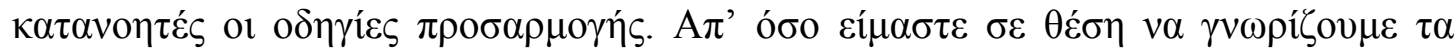

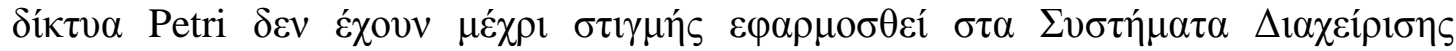

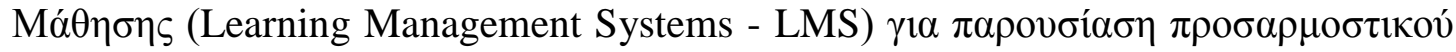

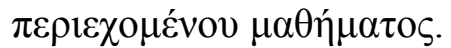

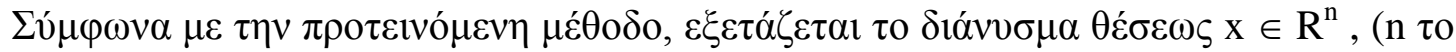

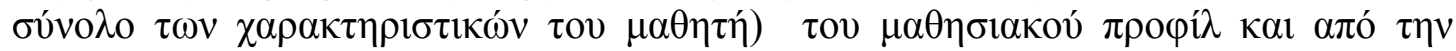

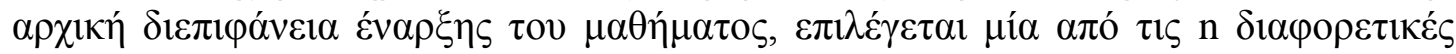

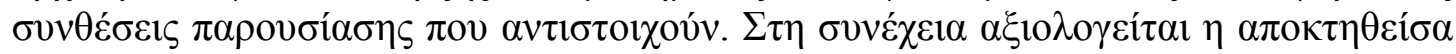

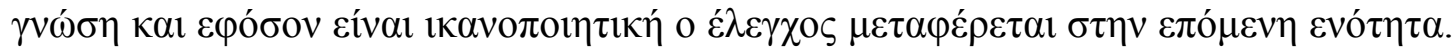

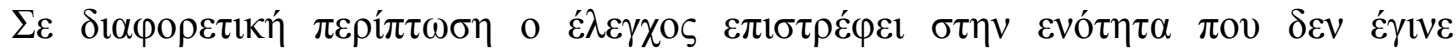

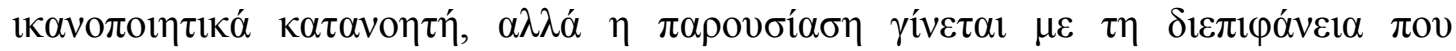

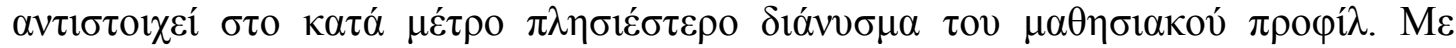

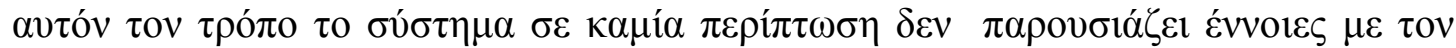

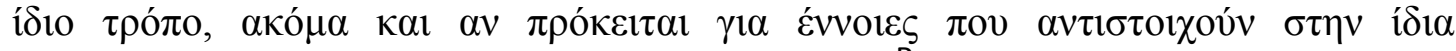

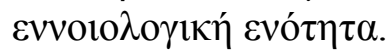

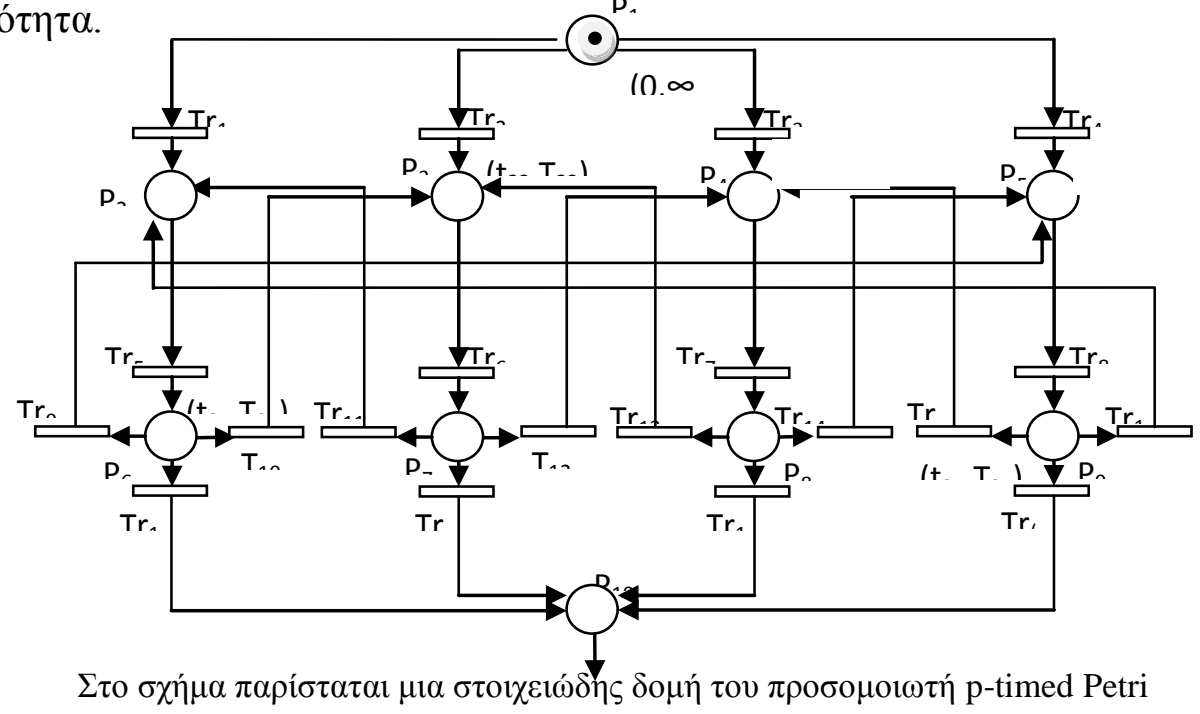

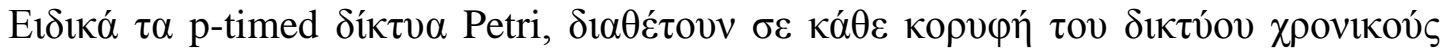

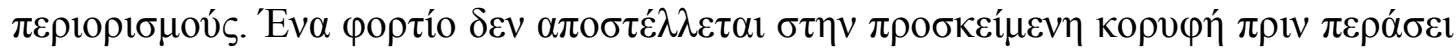

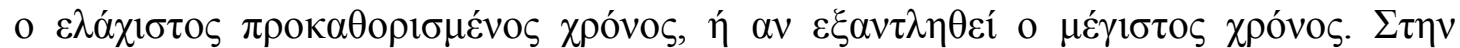

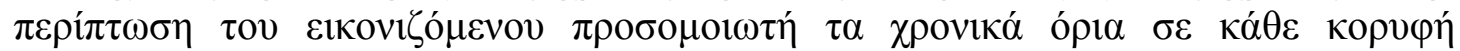

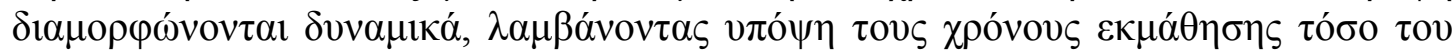

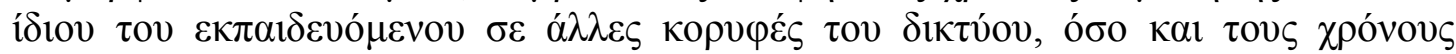




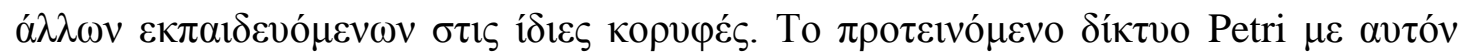

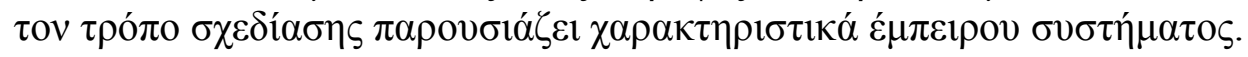

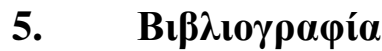

Anagnostopoulos, C.-N from: http://ieeexplore.ieee.org/xpls/abs_all.jsp?arnumber=5298878\&tag=1

AutoTutor from: http://www.informaworld.com/smpp/content $\sim \mathrm{db}=$ all? content $=10.1080 / 08839510802631745$

Barros, B., Verdejo, M.F., Read, T., Mizoguchi, R., (2002). Lecture Notes in Computer Science; vol 2313. Proceedings of the Second Mexican International Conference on Artificial Intelligence: Advances in Artificial Intelligence, Springer-Verlag, London

Boehnera K., DePaulab R., Dourishc P., Sengersa Ph., (2007), How emotion is made and measured, Int. Journal Human Computer Studies, 65, pp. 275-291

Botsios S., Georgiou D.A., (2008). Recent Adaptive e-Learning Contributions Towards A Standard Ready Architecture, MCCSIS08 / IADIS - International Association for Development of the Information Society, 22 to 27 July. Amsterdam, Netherlands

Botsios S., Georgiou D.A., Safouris N., (2007). Learning Style Estimation via Bayesian Networks, Proceedings of WEBIST (2007), Barcelona, Spain

Bunt A., Conati C., (2003). Probabilistic Student Modeling to Improve Exploratory Behavior. In User Modeling and User-Adapted Interaction, vol 13(3)

Classic Reload Editor, from: http://www.reload.ac.uk/new/editor.html, May (2011)

Damasio A. R., (1994). Descartes' error, Grosset/Putnam, New York, 1994

Dickerson J. A., \& Kosko B., (1993). Virtual worlds as fuzzy cognitive maps. Paper read at 1993 IEEE Annual Virtual Reality International Symposium

Dublin Core Initiative Metadata, from: http://dublincore.org/, May (2011)

Dunn, R., Dunn K., Price, G. (1985). Learning Style Inventory Research Manual, Price Systems

Dunn, R., Dunn, K., (1992). Teaching elementary students through their individual learning styles: Practical approaches for grades 3-6, Boston, MA: Allyn \& Bacon

Felder, R., Silverman, L., (1988). Learning and Teaching Styles in Engineering Education. In Engineering Education, vol. 78(7), pp. 674-681

Felder, R., Silverman, L., (1988). Learning and Teaching Styles in Engineering Education. In Engineering Education, vol. 78(7), pp. 674-681

Gao S., Dew R., (2007). Enhancing Web-based Adaptive Learning with Colored Timed Petri Net, Lecture Notes in Computer Science (including subseries Lecture Notes in Artificial Intelligence and Lecture Notes in Bioinformatics), Vol. 4798 LNAI, p.p. 177-185

Gao S., Zhang Z., Wells J., Hawryszkiewycz H.T., (2008). 'Supporting adaptive learning with high level timed Petri nets', International Journal of Intelligent Systems Technologies and Applications, Volume 4, Number 3-4 , pp. 341 - 354

Garcia, P., Amandi, A., Schiaffino, S., Campo, M., (2005). Evaluating Bayesian network's precision for detecting students' learning styles. In Computers and Education (in press)

Georgiou D. A., Makry D., (2004). A Learner's Style and Profile Recognition via Fuzzy Cognitive Map. In. Proceedings of the IEEE International Conference on Advanced Learning Technologies (ICALT04), IEEE

Georgiou D.A., Tsoulouchas G. (2011). Adaptive Management of Learning Content, Proceedings of ED-MEDIA 2011, Lisbon, from http://www.editlib.org/noaccess/38058

Georgiou D.A., Botsios S., Mitropoulou V. , Papaioanou M., Schizas C. and Tsoulouhas G. (2011), Learning Style Recognition Based on an Adjustable Three-Layer Fuzzy Cognitive Map, under review process in Journal of Computers and Education

Georgiou D.A., Germanakos P., Samaras G., Andreou P. and Tsoulouchas G. (2011). Detection of Emotional State Based on hands Motility, under preparation

Georgopoulos, V. C., G. A. Malandraki, \& C. D. Stylios. (2003). A fuzzy cognitive map approach to differential diagnosis of specific language impairment. Artificial Intelligence in Medicine, 29 (3), pp. 261-278

Higashiyama, A., Imoto, H., \& Tsuinashi, S. (2005). Comprehension of emotions accompanied by everyday actions: Comparison of biological-motion pictures with real-person pictures, Shinrigaku Kenkyu, 76(5), pp. 486-491

Holt A., (1968). Information system theory project, Dept.of Commerce, Clearinghouse, Springfield, VA 22151

Iida S., Nakao T., and Ohira H., (2011). Implicit Attenuation of Subsequent Emotion by Cognitive Activity from http://cabn.psychonomic-journals.org/content/8/3/282 
RELOAD project from http://www.reload.ac.uk/

Kaltz, L., Rezaei, R., (2004). Evaluation of the reliability and validity of the cognitive style analysis. In Personality and Individual Differences, vol 36

Khanchandani, K. B., \& Hussain, M. A. (2009) Emotion recognition using multilayer perceptron and generalized feed forward neural network. Journal of Scientific and Industrial Research, 68(5), pp. 367-371. Retrieved from www.scopus.com

Ko, K. -., \& Sim, K. -. (2010). Emotion recognition in facial image sequences using a combination of AAM with FACS and DBN, Retrieved from www.scopus.com

Kolb, D., (1984). Experiential Learning: Experience as the Source of Learning and Development. Prentice Hall, Englewood Cliffs

Kolb, D., (1999). Learning Style Inventory - version 3: Technical Specifications, TRG Hay/McBer, Training Resources Group

Learning Technologies Standarts Committee, from: http://www.ieeeltsc.org:8080/Plone/contactinformation May (2011),

LOC Tool,from: http://www.llas.ac.uk/projects/2770

LOM standarts by IEEE, from

http://ltsc.ieee.org/wg12/(200(20612-Final-LOM-Draft.html, May (2011).

LOTTI project, from: http://arc.tees.ac.uk/lotti/ May (2011)

MERLOT -- Multimedia Educational Resource for Online Learning and Teaching, from http://www.merlot.org/merlot/index.htm

Millán, E., Pèrez-de-la-Cruz J., Suárez, E., (2000). Adaptive Bayesian Networks for Multilevel Student Modelling, Intelligent Tutoring Systems. In Proceedings of the Fifth International Conference, Montreal, Canada

Murata T., (1989). Petri Nets: Properties, Analysis, and Applications, Proceedings of the IEEE, Vol. 77, No. 4, April 1989

Murray, W., (1999). An Easily Implemented Linear - time Algorithm for Bayesian Student Modelling in Multi-level Trees, Artificial Intelligence in Education: Open Learning Environments: New Conceptual Technologies to Support Learning, Exploration and Collaboration, Frontiers in Artificial Intelligence and Applications, vol. 50, pp. 413 - 420

Ogbu J. U., (1992). Understanding Cultural Diversity and Learning, Educational Researcher, 21, No 8. 5- 14+24;

Paiva A., Prada R., Picard R.W., (2007). Editors, Affective Computing and Intelligent Interaction, Springer Verlag, Berlin, Heidelberg

Paulmann S.and Pell M. D., (2010). Contextual influences of emotional speech prosody on face processing: How much is enough? Cognitive, Affective, \& Behavioral Neuroscience 10, \#2, 230-242, (2010) DOI: 10.3758/CABN.10.2.230

Pearl, J., (1988). Probabilistic Inference in Intelligent Systems, Morgan Kaufmann, San Mateo, California

Petri C.A., (1966). Communication with Automata, New York: Griffiss Air Force Base. Tech. Rep. RADC-TR-65-377, vol. 1, Suppl. 1

Reye, J., (1996). Lecture Notes In Computer Science; vol 1086. Proceeding of the Third International Conference on Intelligent Tutoring Systems, Springer-Verlag, London

Reye, J., (2004). Student Modelling based on Belief Networks. In International Journal of Artificial Intelligence in Education, vol 14

Rong from http://ieeexplore.ieee.org/xpls/abs_all.jsp?arnumber=4276418\&tag=1

Rong, J., from http://www.sciencedirect.com/science?_ob=ArticleURL\&_udi=B6VC8-4TTF00J$1 \& \_u s e r=109808 \&$ coverDate $=05 \% 2 \mathrm{~F} 31 \% 2 \mathrm{~F}(2009) \& \_r d o c=1 \&$ fmt $=$ high \&_orig $=$ search \& sort $=\mathrm{d} \& \_d$ docanchor $=\&$ view $=\mathrm{c} \& \_$acct $=$C00005) $9631 \& \_$version $=1 \& \_u r l$ Version $=0 \& \_u s e r i d=1$ 09808\&md5=79c617ee0ecc8261eda89f4aa277c536

Sakr, G. E., Elhajj, I. H., \& Abou-Saad Huijer, H. (2010). Support vector machines to define and detect agitation transition. IEEE Transactions on Affective Computing, Retrieved from www.scopus.com

Shen, L., Wang, M., \& Shen, R. (2009). Affective e-learning: Using "motional" data to improve learning in pervasive learning environment. Educational Technology and Society, 12(2), 176189. Retrieved from www.scopus.com

Simon H., (1979) "Motivational and Emotional Controls of Cognition," Models of Thought, pp. 29-38, Yale University Press

Sloman A., Croucher M., (1981), Why Robots will Have Emotions, Proc. of the Seventh International Conference on Artificial Intelligence, pp. 197-202 
Smith, SE., (2001). The relationship between learning style and cognitive style. In Personality and Individual Differences, vol 30

Van Lehn, K., Martin, J., (1995). Student assessment using Bayesian Nets. In International Journal of Human Computer Studies, vol 42

Wiley, D.A., (2000),. Connecting learning objects to instructional design theory: A definition, a metaphor, and a taxonomy. In D.A. Wiley (Ed.), The Instructional Use of Learning Objects. Bloomington, IN: Association for Educational Communications and Technology

WISC-online from: http://www.wisc-online.com/ May (2011)

Zapata-Rivera, JD., Greer, J., (2004). Inspect able Bayesian student modeling servers in multi-agent tutoring systems. In International Journal of Human-Computer Studies, vol 61 